MID-INFRARED SPECTROSCOPY OF CANDIDATE ACTIVE GALACTIC NUCLEIDOMINATED SUBMILLIMETER GALAXIES

This article has been downloaded from IOPscience. Please scroll down to see the full text article. 2010 ApJ 713503

(http://iopscience.iop.org/0004-637X/713/1/503)

The Table of Contents and more related content is available

Download details:

IP Address: 131.215.220.165

The article was downloaded on 09/04/2010 at 23:52

Please note that terms and conditions apply. 


\title{
MID-INFRARED SPECTROSCOPY OF CANDIDATE ACTIVE GALACTIC NUCLEI-DOMINATED SUBMILLIMETER GALAXIES
}

\author{
K. Coppin ${ }^{1}$, A. Pope ${ }^{2,21}$, K. Menéndez-Delmestre ${ }^{3,22}$, D. M. Alexander ${ }^{4}$, J. S. Dunlop ${ }^{5}$, E. Egami $^{6}$, J. Gabor $^{6}$, Edo Ibar $^{7}$, \\ R. J. Ivison ${ }^{5,7}$, J. E. AustermanN ${ }^{8}$, A. W. Blain ${ }^{9}$, S. C. Chapman ${ }^{10}$, D. L. Clements ${ }^{11}$, L. Dunne ${ }^{12}$, S. Dye ${ }^{13}$, \\ D. Farrah ${ }^{14}$, D. H. Hughes ${ }^{15}$, A. M. J. Mortier ${ }^{5}$, M. J. Page ${ }^{16}$, M. Rowan-Robinson ${ }^{11}$, D. Scott ${ }^{17}$, C. Simpson ${ }^{18}$, IAn Smail ${ }^{1}$, \\ A. M. SWINBANK ${ }^{1}$, M. VACCARI ${ }^{19}$, AND M. S. YUN $^{20}$ \\ ${ }^{1}$ Institute for Computational Cosmology, Durham University, South Road, Durham, DH1 3LE, UK \\ ${ }^{2}$ National Optical Astronomy Observatory, 950 N. Cherry Ave., Tucson, AZ 85719, USA \\ ${ }^{3}$ The Observatories of the Carnegie Institution for Science, 813 Santa Barbara St., Pasadena, CA 91101, USA \\ ${ }^{4}$ Department of Physics, Durham University, South Road, Durham, DH1 3LE, UK \\ ${ }^{5}$ Scottish Universities Physics Alliance (SUPA), Institute for Astronomy, University of Edinburgh, Royal Observatory, Blackford Hill, Edinburgh, EH9 3HJ, UK \\ ${ }^{6}$ Steward Observatory, University of Arizona, 933 N. Cherry Ave., Tuscon, AZ 85721, USA \\ ${ }^{7}$ UK ATC, Science and Technology Facilities Council, Royal Observatory, Blackford Hill, Edinburgh EH9 3HJ, UK \\ ${ }^{8}$ Center for Astrophysics and Space Astronomy, University of Colorado, Boulder, CO 80309, USA \\ ${ }^{9}$ Caltech, 249-17, Pasadena, CA 91125, USA \\ ${ }^{10}$ Institute of Astronomy, University of Cambridge, Madingley Road, Cambridge CB3 0HA, UK \\ ${ }^{11}$ Astrophysics Group, Blackett Laboratory, Imperial College, Prince Consort Rd., London SW7 2BW, UK \\ 12 The School of Physics and Astronomy, University of Nottingham, University Park, Nottingham NG7 2RD, UK \\ ${ }^{13}$ School of Physics and Astronomy, Cardiff University, 5, The Parade, Cardiff CF24 3YB, UK \\ ${ }^{14}$ Astronomy Centre, University of Sussex, Falmer, Brighton BN1 9QH, UK \\ ${ }^{15}$ Instituto Nacional de Astrofísica, Óptica y Electrónica, Apartado Postal 51 y 216, 72000 Puebla, Pue., Mexico \\ ${ }^{16}$ Mullard Space Science Laboratory (MSSL), University College London, Holmbury St. Mary, Dorking, Surrey RH5 6NT, UK \\ ${ }^{17}$ Department of Physics \& Astronomy, University of British Columbia, 6224 Agricultural Road, Vancouver, B.C., V6T 1Z1, Canada \\ ${ }^{18}$ Astrophysics Research Institute, Liverpool John Moores University, Twelve Quays House, Egerton Wharf, Birkenhead CH41 1LD, UK \\ ${ }^{19}$ Department of Astronomy, University of Padova, Vicolo Osservatorio 3, I-35122, Padova, Italy \\ ${ }^{20}$ Department of Astronomy, University of Massachusetts, Amherst, MA 01003, USA \\ Received 2009 November 20; accepted 2010 February 28; published 2010 March 23
}

\begin{abstract}
Spitzer spectroscopy has revealed that $\simeq 80 \%$ of submm galaxies (SMGs) are starburst (SB)-dominated in the mid-infrared. Here we focus on the remaining $\simeq 20 \%$ that show signs of harboring powerful active galactic nuclei (AGNs). We have obtained Spitzer-InfraRed Spectrograph spectroscopy of a sample of eight SMGs that are candidates for harboring powerful AGNs on the basis of IRAC color selection $\left(S_{8 \mu \mathrm{m}} / S_{4.5} \mu \mathrm{m}>2\right.$, i.e., likely power-law mid-infrared spectral energy distributions). SMGs with an AGN dominating ( $\gtrsim 50 \%)$ their midinfrared emission could represent the "missing link" sources in an evolutionary sequence involving a major merger. First of all, we detect polycyclic aromatic hydrocarbon (PAH) features in all of the SMGs, indicating redshifts from 2.5 to 3.4, demonstrating the power of the mid-infrared to determine redshifts for these optically faint dusty galaxies. Second, we see signs of both star formation (from the PAH features) and AGN activity (from continuum emission) in our sample: $62 \%$ of the sample are AGN-dominated in the mid-infrared with a median AGN content of 56\%, compared with $<30 \%$ on average for typical SMGs, revealing that our IRAC color selection has successfully singled out sources with proportionately more AGN emission than typical SB-dominated SMGs. However, we find that only about $10 \%$ of these AGNs dominate the bolometric emission of the SMG when the results are extrapolated to longer infrared wavelengths, implying that AGNs are not a significant power source to the SMG population overall, even when there is evidence in the mid-infrared for substantial AGN activity. When existing samples of mid-infrared AGN-dominated SMGs are considered, we find that $S_{8 \mu \mathrm{m}} / S_{4.5 \mu \mathrm{m}}>1.65$ works well at selecting mid-infrared energetically dominant AGNs in SMGs, implying a duty cycle of $\sim 15 \%$ if all SMGs go through a subsequent mid-infrared AGN-dominated phase in the proposed evolutionary sequence.
\end{abstract}

Key words: galaxies: active - galaxies: evolution - galaxies: high-redshift - galaxies: starburst - infrared: galaxies - submillimeter: galaxies

Online-only material: color figures

\section{INTRODUCTION}

The era some 3 Gyr after the big bang $(z \sim 2-2.5)$ coincides with a peak in activity in two important populations: quasi-stellar objects (QSOs), which represent accretion onto supermassive black holes (SMBHs), and a population of extremely luminous, but highly obscured galaxies (e.g., Chapman et al. 2005; Wall et al. 2008). The bulk of the luminosity of

\footnotetext{
${ }^{21}$ Spitzer Fellow.

22 NSF Astronomy and Astrophysics Postdoctoral Fellow.
}

these obscured galaxies is emitted in the rest-frame far-infrared waveband. As a result of redshifting, they are most directly selected through their emission in the submillimeter (submm) or $\mathrm{mm}$ wavebands, typically in the atmospheric windows around 850 or $1100 \mu \mathrm{m}$, and so are termed submm galaxies (SMGs). The infrared luminosities $\left(L_{\mathrm{IR}}\right)$ inferred for SMGs from their submm emission are highly uncertain, but assuming, as appears to be the case, that they follow the far-infrared-radio correlation for local starburst (SB) galaxies, then their typical luminosities will be $L_{\mathrm{IR}} \simeq 10^{12}-10^{13} L_{\odot}$ (e.g., Kovács et al. 2006; Murphy et al. 2009). Thus, this population may contain some of the most 


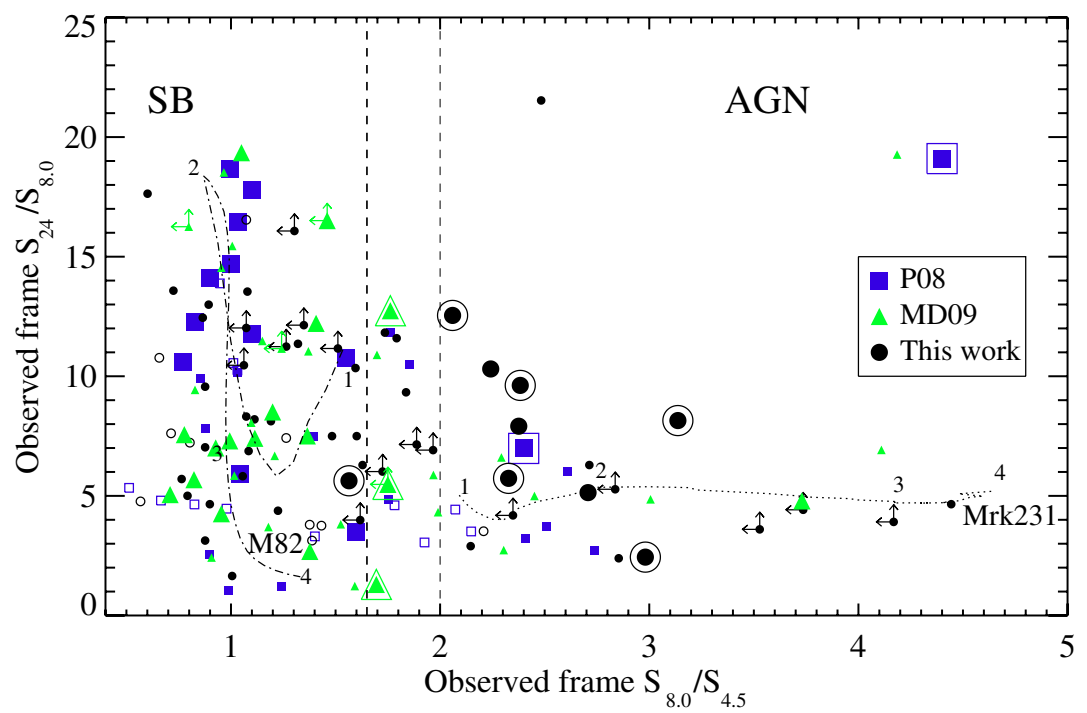

Figure 1. Spitzer color-color diagram as an AGN diagnostic (see, e.g., Ivison et al. 2004; P08). The dotted and dot-dashed curves show the positions of Mrk 231 (an AGN) and M82 (a SB), respectively, as a function of redshift (redshift is indicated by the numbers along the tracks). The smallest filled and open circles represent robust $(P<0.05)$ and tentative $(P>0.05) 24 \mu \mathrm{m} \mathrm{SMG}$ counterparts, respectively, for our parent sample of blank-field SHADES SMGs (Ivison et al. 2007). For comparison, we have also plotted the GOODS-N SMG sample (blue squares; Pope et al. 2006), and also the Chapman et al. (2005) radio-selected spectroscopically confirmed sample of SMGs (green triangles). SMGs from these parent samples that have been observed with the IRS (P08; MD09; this work) are indicated by large versions of the corresponding symbols. Of these IRS-observed SMGs, those with $>50 \%$ contribution from continuum (AGN) emission to the mid-infrared luminosity are circumscribed and tend to lie far from the M82 SB sequence. Although our sample was initially selected on the basis of $S_{8} / S_{4.5}>2$ (thin dashed vertical line), when all the results from the literature are taken into account a selection of $S_{8} / S_{4.5} \gtrsim 1.65$ thus seems to pick out AGNs fairly efficiently, with some small amount of scatter over this boundary (thick dashed vertical line; see Section 4). Extrapolating from this result implies that $\approx 15 \%$ of blank-field SMGs will likely have an enhanced dominant AGN component in the mid-infrared.

luminous galaxies in the universe comparable in luminosity to QSOs.

The increasing availability of precise redshifts for samples of SMGs (e.g., Chapman et al. 2005; Eales et al. 2009) has allowed their properties to be studied in detail. SMGs are strongly clustered (Blain et al. 2004), massive $\left(M_{\star}>10^{11} M_{\odot}\right.$; Borys et al. 2005; Hainline et al. 2009), and gas-rich $\left(f_{\text {gas }} \sim 0.3\right.$; Frayer et al. 1998; Greve et al. 2005; Tacconi et al. 2006, 2008) systems which are known to harbor (apparently) low-luminosity Compton-thin AGNs (i.e., $N_{\mathrm{H}}<10^{24} \mathrm{~cm}^{-2}$; Takata et al. 2006; Alexander et al. 2005b, 2005a, 2008a) and (apparently) strong star formation (SF) activity (SFR $\sim 1000 M_{\odot} \mathrm{yr}^{-1}$; Swinbank et al. 2004; Chapman et al. 2005). Many of these properties, and the similarity between the redshift distributions of QSOs and SMGs (Chapman et al. 2005), support a link between SMGs, QSOs, and the formation phase of massive elliptical galaxies (e.g., Lilly et al. 1999; Archibald et al. 2002; Stevens et al. 2005; Coppin et al. 2008b). In the high-redshift interpretation of the evolutionary sequence first presented by Sanders et al. (1988), SMGs would trace an infrared ultraluminous phase followed by a short "transition phase" where the galaxy would display a mix of SF and obscured AGN activity before evolving into an optically luminous QSO. Studying the relative SF and AGN activity in these "transition" or "missing link" sources and performing a comparison to typical SF SMGs can thus provide important insight on the validity of this evolutionary sequence. Here we focus on the comparison of the mid-infrared spectral properties between existing samples of typical SMGs and a new sample of candidate "transition phase" SMGs. The benefit of undertaking this energy audit in the mid-infrared is that the spectra can provide measurements of both SF and AGN activity simultaneously (see Pope et al. 2008a and Menéndez-Delmestre et al. 2009, hereafter P08 and MD09, respectively), providing a complementary approach to radio (e.g., Ibar et al. 2010) and
X-ray studies of SMGs (e.g., Alexander et al. 2005a; Laird et al. 2010).

The Spitzer InfraRed Spectrograph (IRS; Houck et al. 2004) era enabled the study of the energetics of $\approx 45$ SMGs with $24 \mu \mathrm{m}$ flux densities as low as $\sim 0.1 \mathrm{mJy}$ and with redshifts as high as $z=2.6$, demonstrating that accurate redshifts for dust-enshrouded (and sometimes optically invisible) galaxies can be obtained (e.g., Lutz et al. 2005; Valiante et al. 2007; Menéndez-Delmestre et al. 2007; MD09; P08). The majority of the IRS work has confirmed that SMGs are primarily SBdominated systems, with hot dust continuum from an AGN contributing at most $30 \%$ of the mid-infrared luminosity. Only about $15 \%$ of blank-field SMGs appear to be continuumdominated in the mid-infrared (i.e., $>50 \%$ contribution; P08), with InfraRed Array Camera (IRAC; Fazio et al. 2004) $8.0 \mu \mathrm{m}$ to $4.5 \mu \mathrm{m}$ color ratios of $S_{8} / S_{4.5}>2$. Mid-infrared continuumdominated SMGs are potentially an important sub-population of SMGs representing the "missing link" sources in the proposed evolutionary sequence of Sanders et al. (1988), but being the minority of this luminous population, have not yet been studied in a systematic or statistically robust way. Thus, an important question to address is: are the energetics of these composite $\mathrm{SB} / \mathrm{AGN}$ objects with submm emission dominated by SBs or by AGNs, even when the IRAC colors indicate that an AGN is likely present?

Other relevant IRS samples include near-infrared-selected SBs, X-ray-selected AGNs (Weedman et al. 2006), and Spitzerselected $0.3 \lesssim z \lesssim 3$ ULIRGs (Yan et al. 2007; Sajina et al. 2007; Farrah et al. 2008; Dasyra et al. 2009; Hernán-Caballero et al. 2009). AGN-dominated sources in these samples tend to reside in a distinct parameter space in Spitzer color-color diagrams, which is consistent with color-redshift evolution tracks of well-known local AGNs (see, e.g., Figure 1; Ivison et al. 2004; Ashby et al. 2006; Hainline et al. 2009). The reason 
why $S_{8} / S_{4.5}>2$ should locate mid-infrared AGN-dominated sources is simple: seeing an enhanced $8 \mu \mathrm{m}$ flux density compared with $4.5 \mu \mathrm{m}$ is expected if a source has significant thermal power-law emission from an AGN accretion disk, which can dilute both the polycyclic aromatic hydrocarbons (PAHs) and the H-opacity minimum (1.6 $\mu \mathrm{m}$ stellar bump, which these channels trace). Although some contamination is expected: SFdominated sources can also show enhanced $S_{8} / S_{4.5}$ at $z \gtrsim 4$ (when these channels begin to sample over the peak of stellar photospheric emission) as well as at $z \lesssim 1$ (since these channels are not yet climbing up the rest frame $1.6 \mu \mathrm{m}$ stellar bump). The color cut is thus appropriate for separating mid-infrared SB-and AGN-dominated SMGs from $z \simeq 1-4$, which is well matched to the known redshift distribution of the SMG population.

We have thus selected eight SMGs from the Submillimeter Common-User Bolometer Array (SCUBA; Holland et al. 1999) Half Degree Extragalactic Survey (SHADES; Mortier et al. 2005; Coppin et al. 2006; Austermann et al. 2010) within this relatively unexplored $S_{8} / S_{4.5}>2$ parameter space that are likely harboring AGNs. These were targeted with the IRS in order to determine the relative contribution of power-law/ AGN emission versus PAH/SF emission to their power output, enabling a comparison to similar IRS samples of more typical SF-dominated SMGs. We use these data to obtain independent redshift estimates as well as to test if the IRAC-color criterion is a secure means of pre-selecting SMG counterparts with an enhanced AGN component compared to typical SMGs-the "missing link" sources in the proposed evolutionary sequence we wish to investigate.

This paper is organized as follows. The sample selection, Spitzer-IRS observations, data reduction, and analysis approach are described in Section 2. In Section 3, we present the main results of the IRS spectroscopy, including redshifts, spectral decomposition and AGN classification, and full spectral energy distribution (SED) fits to determine their total infrared luminosities. We discuss the implications that our results and those of other IRS SMG studies have on the role that SMGs play in galaxy evolution in the framework of the proposed evolutionary sequence in Section 4. Finally, our conclusions are given in Section 5.

Here, we discuss the observed "mid-infrared" spectral properties of our SMGs probed by the IRS which, at our source redshifts of $z>2.5$, traces $\sim 4-10 \mu \mathrm{m}$ in the rest frame. All magnitudes in this paper are on the $\mathrm{AB}$ system, unless otherwise stated. We adopt cosmological parameters from the $W M A P$ fits (Spergel et al. 2003): $\Omega_{\Lambda}=0.73, \Omega_{\mathrm{m}}=0.27$, and $H_{0}=71 \mathrm{~km} \mathrm{~s}^{-1} \mathrm{Mpc}^{-1}$.

\section{SAMPLE SELECTION, OBSERVATIONS, DATA REDUCTION, AND ANALYSIS}

\subsection{Sample Selection and Multi-wavelength Properties}

SHADES provides a suitably large parent sample of SMGs from which we can select a representative and large enough sample of SMG AGN candidates. SCUBA surveyed $\sim 0.25 \mathrm{deg}^{2}$ at $850 \mu \mathrm{m}$ to an rms of $\sim 2 \mathrm{mJy}$, uncovering $120 \mathrm{SMGs}>3.5 \sigma$ in the Lockman Hole East (LH) and the equatorial Subaru-XMM Deep Field (SXDF), centered at J2000 R.A. $=2^{\mathrm{h}} 17^{\mathrm{m}} 57^{\mathrm{s}} .5$, decl. $=-5^{\circ} 00^{\prime} 18^{\prime \prime} .5$ and R.A. $=10^{\mathrm{h}} 52^{\mathrm{m}} 26^{\mathrm{s}} .7$, decl. $=57^{\circ} 24^{\prime} 12^{\prime \prime} .6$, respectively (Coppin et al. 2006). The SHADES fields were subsequently mapped to their full $\sim 0.5 \mathrm{deg}^{2}$ coverage by the Astronomical Thermal Emission Camera (AzTEC; Wilson et al. 2008 ) at $1100 \mu \mathrm{m}$ to an rms depth of $\sim 1-1.5 \mathrm{mJy}$, yielding 114
SMGs above 3.6 $\sigma$ (Austermann et al. 2010), a population akin to the well-known submm or SCUBA galaxies (e.g., Chapin et al. 2009). A detailed comparison of the overlap in the SCUBA and AzTEC SHADES catalogs will be presented in M. Negrello et al. (2010, in preparation).

Ivison et al. (2007), Ibar et al. (2009, 2010), and V. Arumugam et al. (2010, in preparation) have identified $1.4 \mathrm{GHz}$ Very Large Array (VLA), 610 MHz Giant Metre-wave Radio Telescope (GMRT), and/or Mid-Infrared Photometer for Spitzer (MIPS; Rieke et al. 2004) $24 \mu \mathrm{m}$ counterparts for $65 \%$ of the SHADES SMGs. Here we select SMGs with statistically robust $24 \mu \mathrm{m}$ counterparts with $S_{24 \mu \mathrm{m}}>0.2 \mathrm{mJy}$ (ensuring that we achieve an adequate signal-to-noise ratio, $\mathrm{S} / \mathrm{N}$, on the continuum in the IRS spectra) and observed IRAC colors of $S_{8} / S_{4.5}>2$ (Dye et al. 2008; Clements et al. 2008; see Figure 1). Our final sample is comprised of eight sources: four SMGs from each of the SHADES SCUBA and AzTEC surveys.

Our selection criteria apply to approximately $15 \%-25 \%{ }^{23}$ of the SHADES SMGs (or equivalently to 7\%-13\% of SMGs with $24 \mu \mathrm{m}$ counterparts). Note that including the "non-robust" $24 \mu \mathrm{m}$ IDs in these estimates gives similar fractions. In addition, deeper radio and $24 \mu \mathrm{m}$ data would likely yield identifications for all of the SMGs, and there is no evidence in support of SMGs fainter than $200 \mu \mathrm{Jy}$ at $24 \mu \mathrm{m}$ being fundamentally different than perhaps lying at slightly higher redshifts (e.g., Pope et al. 2006). Thus, the IRAC color-selected subset of SMGs studied here should be representative of $\sim 15 \%$ of the blank-field SMG population. We discuss the possibility of more subtle selection effects in Section 4.1.

We also observed a $24 \mu \mathrm{m}$ counterpart (LOCK850.41-2) of an interesting SMG with multiple robust radio and MIPS counterparts, where the other MIPS counterpart (LOCK850.41-1) was previously observed by MD09. We present these data separately in the Appendix, since this source does not make the original color-cut criterion for our sample.

We make use of additional multi-wavelength data sets available for the $24 \mu \mathrm{m}$ SHADES SMG counterparts in order to further interpret our results in the wider context of the entire SMG population and other high-redshift galaxy populations. Available optical-to-radio photometry is given in Table 2 and was retrieved from Dye et al. (2008) and Furusawa et al. (2008): Subaru $B R i^{\prime} z$; Lawrence et al. (2007): $J$ and $K$ from the DR3 UKIRT Infrared Deep Sky Survey release-UKIDSS; and J. S. Dunlop et al. (2010, in preparation): 3.6-8 $\mu \mathrm{m}$ Spitzer-IRAC from the Spitzer Ultra Deep Survey-SpUDS.

The SHADES fields also possess moderately sensitive XMMNewton imaging with exposures of $673 \mathrm{ks}$ in the LH (Brunner et al. 2008), and 18-83 ks in the SXDF (Ueda et al. 2008). In the deeper X-ray data, even X-ray non-detections, when combined with IRS data, can be sufficient to imply 1-2 orders of magnitude of obscuration at rest frame $2-10 \mathrm{keV}$ and can identify Comptonthick AGNs. Given the combined positional uncertainties of the $24 \mu \mathrm{m}$ and X-ray sources (yielding a combined positional uncertainty of $3^{\prime \prime}-4^{\prime \prime}$ ), we search for X-ray counterparts to the SMGs using a $4^{\prime \prime}$ search radius. For any X-ray detections, we extract a $0.5-2 \mathrm{keV}$ flux and calculate the rest-frame $2-10 \mathrm{keV}$ $\mathrm{X}$-ray luminosity, assuming a spectral slope of $\Gamma=1.4$ (the average X-ray spectral slope for sources of this approximate X-ray flux; see Figure 8 of Alexander et al. 2001) to make a small $K$-correction $(\lesssim 30 \%)$. For the X-ray non-detections, we

\footnotetext{
23 The range in this fraction merely represents the fact that several of the SMGs are undetected $8 \mu \mathrm{m}$ and so they could lie on either side of the IRAC color-cut boundary in Figure 1.
} 
Table 1

Spitzer-IRS Observations of SHADES SMGs in the Lockman Hole ("LOCK") and the Subaru-XMM Deep Field ("SXDF")

\begin{tabular}{|c|c|c|c|c|c|c|}
\hline \multirow[t]{2}{*}{ IAU Name } & \multirow[t]{2}{*}{ Nickname } & \multicolumn{2}{|c|}{$24 \mu \mathrm{m}$ Position $(\mathrm{J} 2000)$} & \multicolumn{3}{|c|}{ Integration Time } \\
\hline & & R.A. & Decl. & $\operatorname{LL} 2(\times 120 \mathrm{~s})$ & $\operatorname{LL1}(\times 120 \mathrm{~s})$ & Total (hr) \\
\hline SHADES J105159+572423 & LOCK850.41-2 & 105159.81 & 572425.1 & $3 \times 6$ & $2 \times 6$ & 1.0 \\
\hline AzTEC J105211.61+573510.7 & AzLOCK.62 & 105211.85 & 573510.5 & $14 \times 6$ & $10 \times 6$ & 4.8 \\
\hline AzTE CJ105201.98+574049.3 & AzLOCK.01 & 105201.92 & 574051.5 & $2 \times 6$ & $2 \times 6$ & 0.8 \\
\hline SHADES J021803-045527 & SXDF850.02 & 021803.54 & -045526.9 & $14 \times 6$ & $11 \times 6$ & 5.0 \\
\hline AzTEC J105406.44+573309.6 & AzLOCK.10 & 105406.83 & 573309.1 & $3 \times 6$ & $2 \times 6$ & 1.0 \\
\hline SHADES J105319+572110 & LOCK850.15 & 105319.26 & 572108.3 & $9 \times 6$ & $7 \times 6$ & 3.2 \\
\hline SHADES J021724-045839 & SXDF850.37 & 021724.41 & -045842.0 & $31 \times 6$ & $30 \times 6$ & 12.2 \\
\hline AzTEC J105403.76+572553.7 & AzLOCK.05 & 105403.75 & 572553.5 & $20 \times 6$ & $16 \times 6$ & 7.2 \\
\hline SHADES J105201+572443 & LOCK850.01 & 105201.30 & 572446.1 & $24 \times 6$ & $20 \times 6$ & 8.8 \\
\hline Total time on-source & & & & & & 44.0 \\
\hline
\end{tabular}

have derived $3 \sigma$ upper limits. The X-ray fluxes and flux upper limits are given in Table 3.

It happens that spectroscopic redshifts have not been obtained for the majority of the SMG sample, undoubtedly due to the faintness of the corresponding optical counterparts (see Table 2). All of our SMGs are $R_{\mathrm{AB}} \gtrsim 24$ (except for AzLOCK.10 which is $R_{\mathrm{AB}}=22.65$, and which has not yet been attempted in a spectroscopic follow-up program, to our knowledge), for which Chapman et al. (2005) find an increased spectroscopic redshift failure rate. Therefore, it is not a surprise that only one of our targets has a published tentative optical spectroscopic redshift available for comparison with the IRS-derived redshift (see the Appendix).

\subsection{Spitzer-IRS Observations}

The Spitzer-IRS observations (PID 50183) were taken in spectral mapping mode, with the target being placed at six positions (separated by $20^{\prime \prime}$ ) along the slit using multiple cycles of the longest ramp setting of $120 \mathrm{~s}$ to maximize $\mathrm{S} / \mathrm{N}$ in unit time, while ensuring internal robustness against cosmic rays and the identification of rogue pixels (see Teplitz et al. 2007). We observed the SMGs using Long-Low 1 (LL1; 19.5-38.0 $\mu \mathrm{m}$ ) and Long-Low 2 (LL2; 14.0-21.3 $\mu \mathrm{m}$ ) to ensure that the spectra cover a significant fraction of the full range of PAHs (from 6-17 $\mu \mathrm{m}$ in the rest frame). Integration times were calculated on a source-by-source basis using the observed $24 \mu \mathrm{m}$ fluxes to yield an $\mathrm{S} / \mathrm{N}$ of $>3-4$ in the continuum near the band centeres (i.e., similar quality spectra to those in P08 and MenéndezDelmestre et al. 2007; MD09). We used high-accuracy blue IRS peak-up acquisition on nearby isolated moderately bright Two Micron All Sky Survey stars (2MASS; Skrutskie et al. 2006). To verify the calibration in the LL2 spectra we also obtained $16 \mu \mathrm{m}$ IRS peak-up imaging with $2 \times 30 \mathrm{~s}$ cycles in a five-point dither for each target for an additional $1.7 \mathrm{hr}$ in total. Table 1 gives our target list and integration times for the spectroscopy. Our observations were obtained in 2008 May, June (LH targets), and September (SXDF targets) in a total of $58.4 \mathrm{hr}$.

\subsection{Data Reduction}

We begin with the Spitzer pipeline (Version S18) basic calibrated data. In reducing the IRS data, we follow the same approach used to reduce IRS spectra of faint sources outlined in P08). To summarize, we identify and clean rogue pixels in the two-dimensional (2D) files, fit and subtract latent build-up on the arrays, perform sky subtraction by creating a normalized "supersky" for each Astronomical Observation Request (AOR) in which all other sources have been masked, and co-add all the 2D sky-subtracted data files for each nod position. We extract the 1D spectrum from the 2D co-added files for each nod using the optimal-extraction mode in SPICE. ${ }^{24}$ Along with the target spectrum we also extract a residual sky spectrum from each 2D co-add file, which we use to determine uncertainties on our final 1D spectra. We trim all the spectra to $<35.0 \mu \mathrm{m}$ in order to restrict the analysis to the reliable and least noisy portion of the data. The observed spectra are shown in Figure 4.

We process the IRS $16 \mu \mathrm{m}$ peak-up images (PUIs) with the SSC pipeline S18. We then correct for latent charge accumulation in the PUIs by subtracting the mode flux of the central $30 \times 45$ pixels from each individual exposure. We eliminate residual sky background by subtracting from each science exposure a median sky, created from median-collapsing all charge-corrected exposures and scaled to match off-source background level for each exposure. All sky-subtracted, chargecorrected science exposures are mosaicked into a single image using the standard MOPEX mosaicking pipeline, keeping the native PUI resolution scale of $1^{\prime \prime} .8$ pixel $^{-1}$. We derive $16 \mu \mathrm{m}$ photometry by performing point-response function (PRF) fitting analysis with the MOPEX/APEX single-frame pipeline, relying on the blue PUI PRF. ${ }^{25}$ We use "peak" thresholding as part of the image segmentation algorithm, setting the detection threshold to a $2 \sigma$ level within a $5 \times 5$ pixel fitting area to ensure adequate detection of faint sources. To characterize the uncertainties in the derived integrated fluxes, we rely on the integrated noise within an on-source $5 \times 5$ pixel aperture in the uncertainty mosaic. The $16 \mu \mathrm{m}$ imaging photometry is reported in Table 2 and shown in Figure 2.

We verify the calibration of our final 1D spectra by comparing to the MIPS $24 \mu \mathrm{m}$ and IRS/PUI $16 \mu \mathrm{m}$ fluxes. In all cases the spectra are consistent with the imaging photometry within the uncertainties.

\subsection{Analysis}

Our aim is to determine if our mid-infrared spectra show signs of AGN dominance, and if so, to then compare these spectral properties with the overall SMG population. We have thus followed a similar analysis approach to P08 in order to facilitate a direct unbiased comparison with existing SpitzerIRS samples of SMGs. We have organized the paper so that the following subsections contain all the salient details of our analysis approach, with the results presented separately

\footnotetext{
${ }^{24}$ Available at http://ssc.spitzer.caltech.edu/postbcd/spice.html.

25 Available at http://ssc.spitzer.caltech.edu/irs/calibrationfiles/peakuppsfprf/.
} 
Table 2

Optical-to-Radio Photometry of the SMG $24 \mu \mathrm{m}$ Counterparts

\begin{tabular}{|c|c|c|c|c|c|c|c|c|c|c|c|c|c|c|c|c|}
\hline Nickname & $\overline{B B}$ & 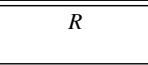 & $\overline{\overline{i^{\prime}}}$ & $\overline{z z}$ & 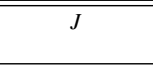 & $\bar{~} \bar{K}$ & $\begin{array}{c}3.6 \mu \mathrm{m} \\
(\mu \mathrm{Jy})\end{array}$ & $\begin{array}{c}\begin{array}{c}4.5 \mu \mathrm{m} \\
(\mu \mathrm{Jy})\end{array} \\
{ }^{2}\end{array}$ & $\begin{array}{c}5.8 \mu \mathrm{m} \\
(\mu \mathrm{Jy})\end{array}$ & $\begin{array}{l}8 \mu \mathrm{m} \\
(\mu \mathrm{Jy})\end{array}$ & $\begin{array}{c}16 \mu \mathrm{m} \\
(\mu \mathrm{Jy})\end{array}$ & $\begin{array}{c}24 \mu \mathrm{m} \\
(\mu \mathrm{Jy})\end{array}$ & $\begin{array}{c}\begin{array}{c}850 \text { or } 1100 \mu \mathrm{m} \\
(\mathrm{mJy})\end{array} \\
\end{array}$ & $\begin{array}{c}1.4 \mathrm{GHz} \\
(\mu \mathrm{Jy})\end{array}$ & $\bar{c} \alpha_{1.4}^{350}$ & $\alpha_{610}^{1.4}$ \\
\hline AzLOCK.01 & & & & & $22.84 \pm 0.10$ & $21.12 \pm 0.04$ & $42.1 \pm 4.5$ & $62.1 \pm 5.8$ & $2362.2 \pm 79.9$ & $194.8 \pm 14.3$ & $697.5 \pm 51.5$ & $1587.3 \pm 43.6$ & $6.6_{-1.0}^{+0.9}$ & $258 \pm 11$ & $0.69 \pm 0.03$ & $1.01 \pm 0$ \\
\hline XDF850.02 & 26.12 & 25.33 & 25.26 & 24.96 & 24.40 & 22.54 & $17.0 \pm 0.8$ & $22.5 \pm 0.8$ & $<48$ & $60.9 \pm 4.5$ & $<171.0$ & $313.0 \pm 47.0$ & $10.2_{-1.6}^{+1.0}$ & $66 \pm 11$ & $0.91 \pm 0.06$ & \\
\hline AzLOCK.10 & $23.04 \pm 0.01$ & $22.65 \pm 0.01$ & $22.40 \pm 0.01$ & $22.23 \pm 0.01$ & $20.87 \pm 0.06$ & $20.16 \pm 0.02$ & $39.7 \pm 4.4$ & $50.1 \pm 5.2$ & $71.0 \pm 14.0$ & $116.6 \pm 11.1$ & $269.1 \pm 46.1$ & $668.4 \pm 29.5$ & $4.1_{-1.0}^{+0.9}$ & $77 \pm 9$ & $0.83 \pm 0.06$ & $-1.32 \pm 0.2$ \\
\hline ОСК850.15 & $26.54 \pm 0.12$ & $02 \pm 0.05$ & $25.24 \pm 0.09$ & $50 \pm 0$ & $>22.33$ & $22.25 \pm 0.12$ & $9.7 \pm 2.2$ & $15.4 \pm 2.9$ & $<18$ & $36.7 \pm 6.5$ & $105.4 \pm 46.0$ & $353.0 \pm 20.0$ & $13.3_{-5.0}^{+4.3}$ & $105 \pm 6$ & $0.88 \pm 0.09$ & $-1.10 \pm 0$ \\
\hline SXDF850.37 & $27.82 \pm 0.26$ & $26.87 \pm 0.16$ & $26.96 \pm 0.20$ & $26.49 \pm 0.32$ & $25.20 \pm 0.79$ & $23.25 \pm 0.21$ & $14.8 \pm 0.6$ & $25.2 \pm 0.9$ & $<48$ & $75.0 \pm 5.2$ & $138.0 \pm 53.8$ & $183.0 \pm 47.0$ & $4.6_{-2.6}^{+2.20}$ & $41 \pm 9$ & $0.85 \pm 0.15$ & \\
\hline AzLO & $25.94 \pm 0.07$ & $25.46 \pm 0.08$ & $25.85 \pm 0.16$ & $25.18 \pm 0.21$ & $>22.33$ & $>22.93$ & $8.9 \pm 2.1$ & $12.5 \pm 2.6$ & $<18$ & \pm 5.9 & & & & $138 \pm 9$ & $0.75 \pm 0.05$ & -0.84 \\
\hline LOCK850.01 & $>27.17$ & $25.83 \pm 0.11$ & $25.35 \pm 0.10$ & $24.89 \pm 0.16$ & $>22.33$ & $>22.93$ & $5.1 \pm 1.6$ & $8.4 \pm 2.2$ & $12.2 \pm 2.2$ & $17.3 \pm 4.7$ & $<125.7$ & $217.0 \pm 16.0$ & $8.9_{-1.0}^{+1.0}$ & $110 \pm 6$ & $0.79 \pm 0.03$ & $-0.05 \pm 0$ \\
\hline
\end{tabular}

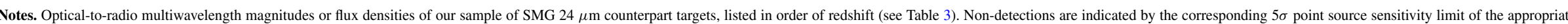

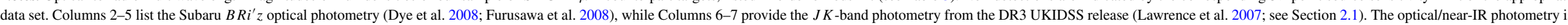

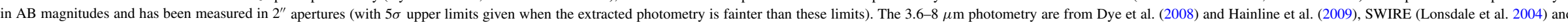

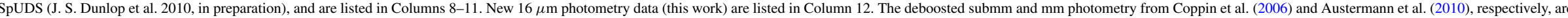

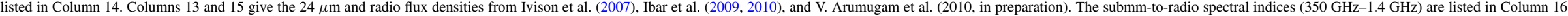

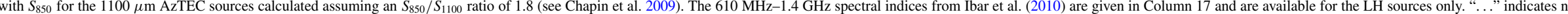
coverage at the source position in this band. 
Table 3

Mid-Infrared and X-ray Derived Properties

\begin{tabular}{lccccc}
\hline \hline \multicolumn{1}{c}{ SMG ID } & $z_{\text {IRS }}$ & $\begin{array}{c}\text { Continuum } \\
(\%)\end{array}$ & $\begin{array}{c}v L_{6 \mu \mathrm{m}} \\
\left(10^{45} \mathrm{erg} \mathrm{s}^{-1}\right)\end{array}$ & $\begin{array}{c}S_{\mathrm{X}}(0.5-2 \mathrm{keV}) \\
\left(10^{-16} \mathrm{erg} \mathrm{cm}^{-2} \mathrm{~s}^{-1}\right)\end{array}$ & $\begin{array}{c}L_{\mathrm{X}}(2-10 \mathrm{keV}) \\
\left(10^{43} \mathrm{erg} \mathrm{s}^{-1}\right)\end{array}$ \\
\hline AzLOCK.62 & $2.48 \pm 0.03$ & 32 & 0.416 & $<5.23$ & $<3.55$ \\
AzLOCK.01 & $2.50 \pm 0.02$ & 71 & $6.76(7.21)$ & $<9.07$ & $<6.31$ \\
SXDF850.02 & $2.55 \pm 0.06$ & 10 & 0.0374 & $<10.95$ & $<7.81$ \\
AzLOCK.10 & $2.56 \pm 0.03$ & 77 & 2.96 & $<.4$ & 3.19 \\
LOCK850.15 & $2.76 \pm 0.01$ & 62 & 1.94 & $<6.08$ & $<5.16$ \\
SXDF850.37 & $2.78 \pm 0.04$ & 51 & 0.641 & $<6.94$ & $<13.0$ \\
AzLOCK.05 & $2.82 \pm 0.02$ & 26 & $0.378(1.84)$ & $<4.78$ & $<6.15$ \\
LOCK850.01 & $3.38 \pm 0.02$ & 56 & 1.29 & $<.94$ \\
\hline
\end{tabular}

Notes. In Column 2, we list the spectroscopic redshifts derived from the fitting procedure described in Section 2.4.1 for the PAH features in the mid-infrared spectra. In Column 3, we give the AGN contribution to the mid-infrared, expressed as a percentage of the total mid-infrared luminosity covered by the IRS spectra, as derived from the continuum power-law fits from Section 2.4.2. Column 4 gives the rest-frame $6 \mu \mathrm{m}$ luminosities based on the best-fitting power-law component, assuming that the AGN contributes to all of the emission at $6 \mu \mathrm{m}$ (with the extinction-corrected values given in parentheses where appropriate). Column 5 gives the observed-frame X-ray fluxes of the detection and $3 \sigma$ upper limits for the non-detections, and Column 6 gives the corresponding rest-frame $\mathrm{X}$-ray luminosities, calculated using the X-ray flux and the IRS-derived redshifts; $\Gamma=1.4$ is used to make small $K$-corrections (see Section 2.1).

a The counterpart is $1^{\prime \prime}$ away from the $24 \mu \mathrm{m}$ position.

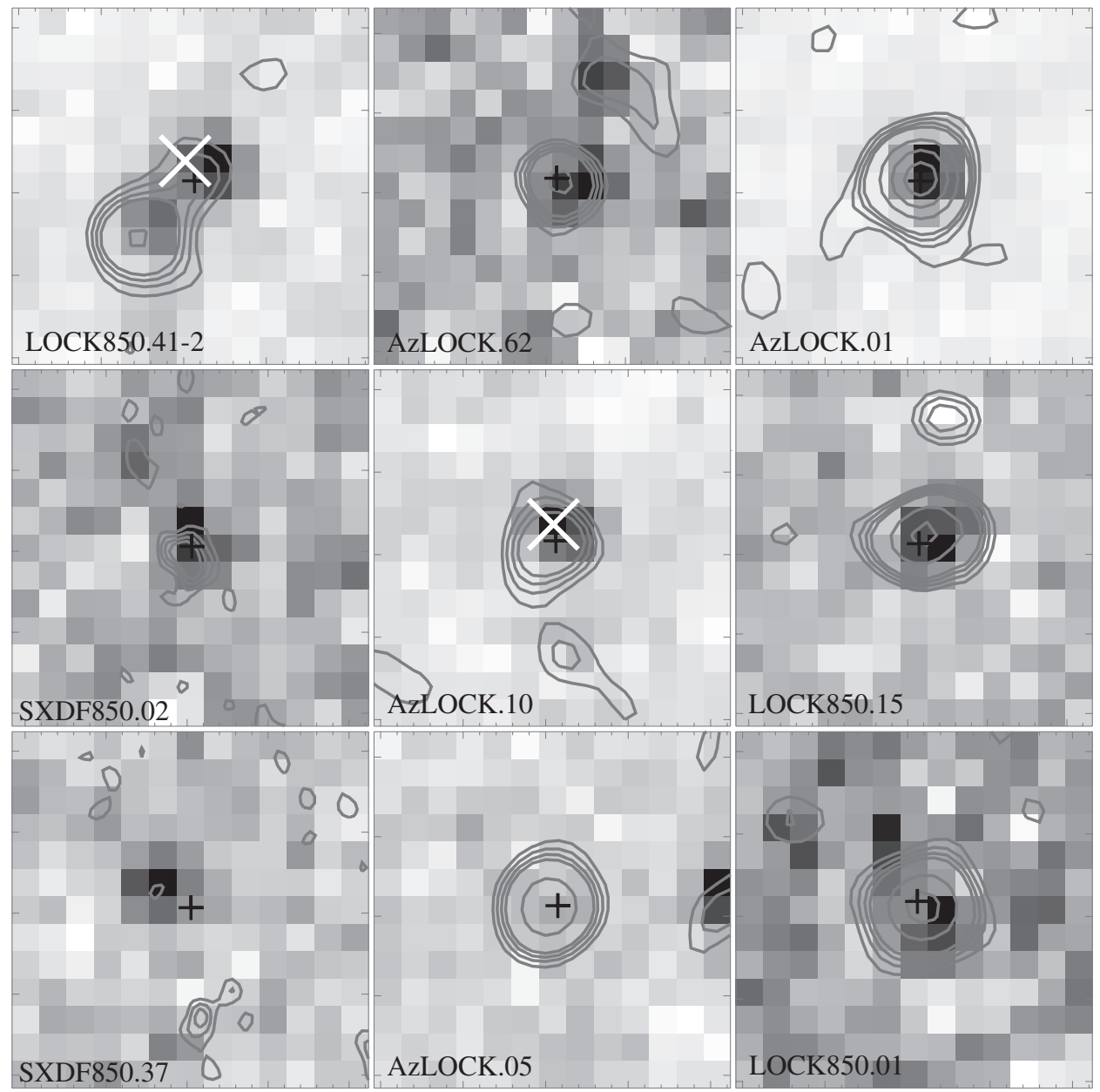

Figure 2. New $16 \mu \mathrm{m} \mathrm{20} 0^{\prime \prime} \times 20^{\prime \prime}$ imaging (roughly twice the size of the aperture of the IRS observations) centered on the $24 \mu \mathrm{m}$ positions of the nine SMG targets (in order of increasing redshift; see Table 3; left-right/top-bottom), with VLA $2 \sigma, 3 \sigma, 4 \sigma, 5 \sigma, 10 \sigma, 15 \sigma$, and $20 \sigma$ contours overlaid. Source names are given in each panel (see Table 1). The sample has been selected on the basis of being $24 \mu \mathrm{m}$ detected and IRAC color-tuned in order to pick out potential AGN-dominated SMGs, although consequently the majority of the targets also appear to be radio-bright, which is consistent with an AGN-biased sample. XMM-Newton detections are shown by the "X" symbols, and only exist for the minority of the sample-deeper X-ray data would likely yield a higher detection fraction, unless the sources are Compton-thick (see the text).

in Section 3. In summary, in Section 2.4.1, we identify the PAH features present in our spectra and determine redshifts. We then decompose each individual spectrum into SF and
AGN components in Section 2.4 .2 in order to classify the fractional contribution of AGNs to the mid-infrared luminosity. In Section 2.4.3, we extrapolate our results to determine the 


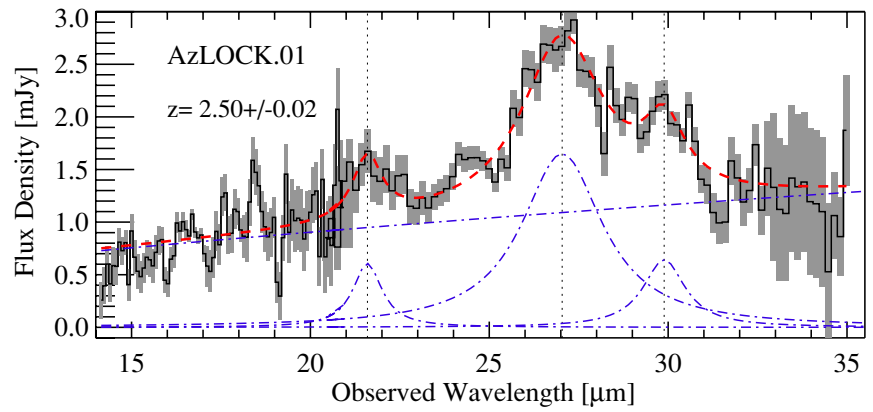

Figure 3. Example of the spectral line decomposition of an SMG IRS spectrum, used to compute the redshift for the source and to measure the line profile features (see Section 2.4.1). The solid black curve is the raw (unsmoothed) IRS spectrum, while the shaded region represents the associated $1 \sigma$ noise from the sky background. The (red) dashed curve shows the best-fit SED to the mid-infrared spectrum, comprising a strong continuum component ((blue) dotdashed power-law) and PAH features ((blue) dot-dashed Lorentzian profiles) at $6.62,7.71$, and $8.61 \mu \mathrm{m}$ (indicated by vertical lines).

(A color version of this figure is available in the online journal.)

full mid-to-far-infrared SEDs in order to estimate the AGN contribution to the bolometric luminosity of these systems.

\subsubsection{Redshift Determination and Spectral Line Measurements}

In order to derive the source redshifts, we simultaneously fit a power-law continuum component and Lorentzian profiles to the spectra using a Levenberg-Marquardt least-squares method, including the residual sky errors in the fit determination (e.g., Sajina et al. 2007), assuming the following rest-frame wavelengths for the main PAH features that are visible: 6.22, 7.71, and $8.61 \mu \mathrm{m}$ (Draine \& Li 2007). We do not impose any joint constraints on the line ratios or centers (i.e., to all lie at the same redshift), as we aim to obtain individual redshift estimates from each PAH feature. See Figure 3 for an example of these fitting results. Our fitting routine returns the formal $1 \sigma$ statistical error estimates for each set of best-fitting parameters derived from the square root of the diagonal elements of the covariance matrix, and we adopt these errors in the ensuing analysis. We verify that these formal errors are reasonable through Monte Carlo simulations similar to those described in P08.

The final redshift for each SMG is calculated by taking a weighted average of the individual redshift estimates from the visible PAH lines (although we neglect the $8.61 \mu \mathrm{m}$ PAH feature in the redshift estimate because it appears relatively weak and significantly blended with the much stronger neighboring $7.71 \mu \mathrm{m}$ feature). The total error on the redshift is calculated from $1 \sigma=\left(\sum_{i=1}^{n} \sigma_{\mathrm{PAH}_{\mathrm{i}}}^{-2}\right)^{-1 / 2}$ with an additional "centroiding error" (calculated as the maximum spread in the line centering from the Monte Carlo simulations) added in quadrature. For sources with only one PAH line, the $1 \sigma$ redshift error is taken to be the centroiding error derived from the simulations (the more conservative choice). Note that the redshift errors range from 0.01 to 0.05 , consistent with the similar quality spectra in P08 and MD09. For comparison, we use the Brandl et al. (2006) SB template with fixed PAH line widths and positions and find that this gives redshift estimates consistent with our method (which is essentially equivalent to performing a combined fit of all the lines simultaneously, but with a more accurate measure of the uncertainty of the redshift).

Once redshifts have been obtained (see Table 3), we calculate the rest-frame PAH line equivalent widths (EWs) and luminosities from our Lorentzian model fits, propagating through the redshift and parameter errors from the fits (see Table 4).

\subsubsection{Determining the Relative AGN and SF Contributions in the Mid-Infrared}

We determine the relative AGN and SF contributions by fitting each spectrum with a model comprised of three main components (extinction, PAHs, and a continuum power law) of the form $F_{v}=c_{0} v^{-c_{1}} e^{-c_{3} \tau_{v}}+c_{2} f_{v, \mathrm{M} 82}$ using a $\chi^{2}$ minimization approach (see P08). ${ }^{26}$ The AGN emission is characterized by a power law with both the normalization and slope as free parameters, and $\tau_{9.7}$ is obtained from the Draine (2003) extinction curves. We assume that all of the PAH line emission is powered by a SB (e.g., Rigopoulou et al. 1999; Laurent et al. 2000), and the SB/PAH emission is fitted using the midinfrared spectrum of M82 from Förster Schreiber et al. (2003), which is known to fit and describe the mid-infrared spectra of the SB-dominated SMG population well (e.g., P08). We derive the fraction of the mid-infrared luminosity that comes from the continuum component by integrating the continuum portion of the best-fitting model and dividing by the area under the total fit. The use of different templates was explored by Sajina et al. (2007) and P08 (e.g., using NGC 7714 in place of M82), and these authors find that it makes little difference on the final outcome of the decomposition, especially given the small wavelength range covered by the spectra. In addition, we perform the decomposition using the Brandl et al. (2006) SB template in place of M82 and find that they yield comparable fractions.

\subsubsection{Full SED Fitting and Determination of $L_{\mathrm{IR}}$}

One of the main goals is to determine if our sample of SMG mid-to-far-infrared SEDs resemble that of typical SMGs or if an additional AGN component is required to fit the data. With this goal in mind, using the same simple templates and approach as P08 will enable a direct and fair comparison between the two samples. We have thus used the full mid-infrared-to-radio composite SED from P08, comprised of their mid-infrared SMG SB composite spectrum spliced with the best-fitting modified Chary \& Elbaz (2001, CE01 hereafter) template, to represent the SF component, and we have used the SED of Mrk 231, consisting of its IRS spectrum (Armus et al. 2007) spliced together with the CE01 template that best fits the far-infrared/submm photometry of Mrk 231, to represent the AGN component. Although the resulting decomposition will thus depend heavily on the templates we have assumed, these choices will allow us to determine the relative differences between the spectral decomposition of our sample of AGN-dominated SMGs and of more typical SB-dominated SMGs from P08. Nevertheless, we discuss and explore other choices for the SB and AGN templates in Section 3.4 to verify the dependence of our results on the assumed templates.

We fit linear combinations of these templates to the data, including the mid-infrared IRS spectra, the 850 or $1100 \mu \mathrm{m}$ photometry, and, where available, $350 \mu \mathrm{m}$ photometry from Coppin et al. (2008a) using a Levenberg-Marquardt leastsquares approach. We also verify that available 70 and $450 \mu \mathrm{m}$ $3 \sigma$ upper limits from Hainline et al. (2009) and Coppin et al. (2006), respectively, are not violated in the final fits. We then integrate the total best-fitting linear combination of the SB and AGN SED components in the rest-frame from 8 to $1000 \mu \mathrm{m}$ to obtain the total infrared luminosities, $L_{\mathrm{IR}}$, and calculate the

\footnotetext{
26 In contrast to the more typical SMGs from P08, we found that the fitting never required additional extinction of the PAH component, so this term has been omitted from our model (cf. Equation (1) in P08).
} 

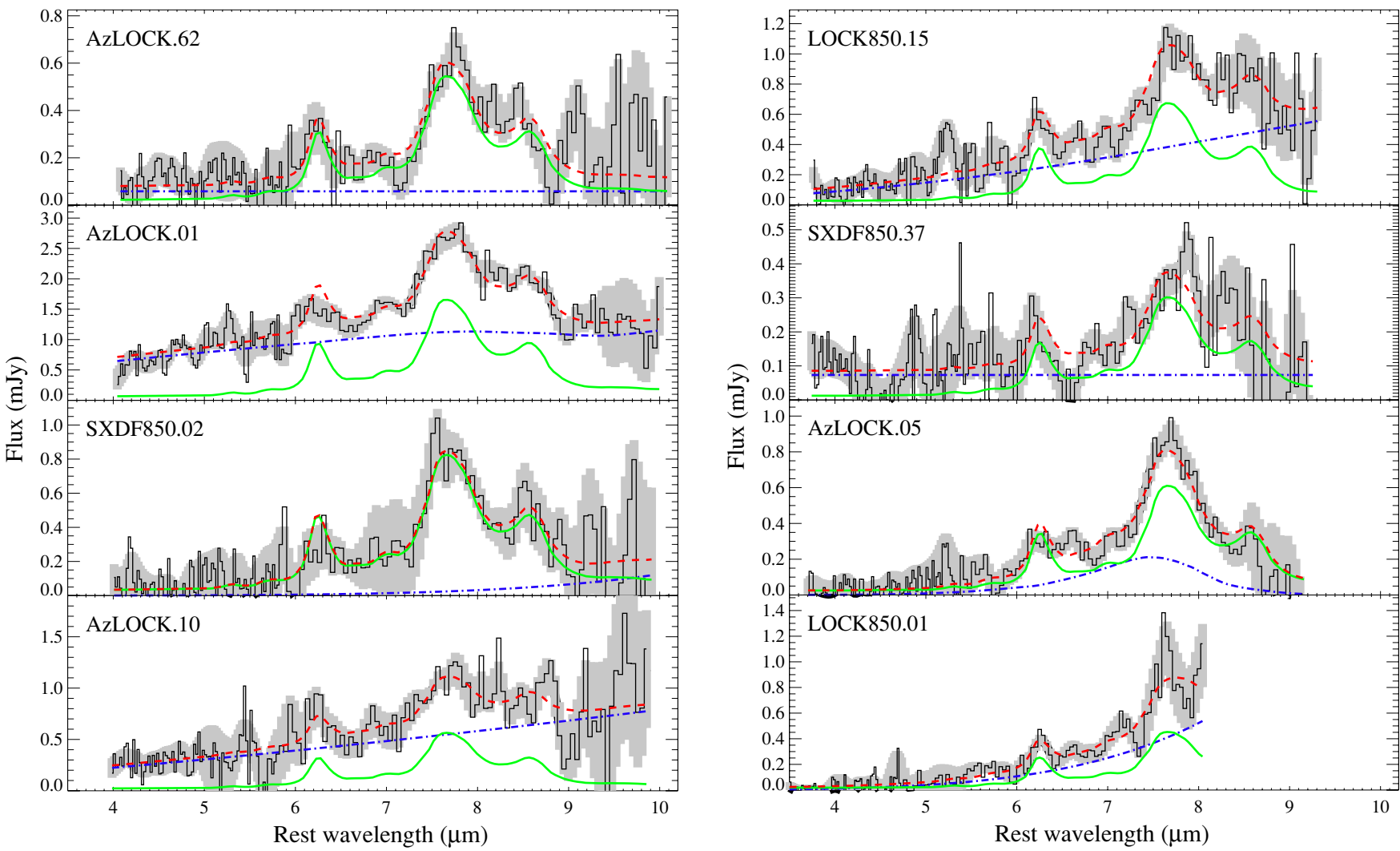

Figure 4. IRS spectra of our AGN-candidate sample of SMGs, listed in order of increasing redshift and shifted to rest-frame wavelengths in order to facilitate an easy inter-comparison of the spectral features. Source names are given in each panel (see Table 1). The solid black curves are the raw (unsmoothed) IRS spectra, while the shaded region represents the associated $1 \sigma$ noise from the sky background (see Section 2.3). The red dashed line is the best-fitting model (see Section 2.4.2), which is composed of an M82 PAH template (green line) and a power-law component (blue dot-dashed line) with extinction applied. The continuum (AGN) fraction for each source is calculated from the spectral decomposition (see Table 3). The spectra and fitting reveal that our sample of candidate AGN-dominated SMGs all show PAH features atop a range of mid-infrared continuum slopes, indicating a mix of SB and AGN properties in the sample.

Table 4

PAH Luminosities, Equivalent Widths, and Infrared Luminosities

\begin{tabular}{|c|c|c|c|c|c|c|c|c|c|}
\hline \multirow[t]{2}{*}{ SMG ID } & \multicolumn{3}{|c|}{ PAH Luminosity $\left(10^{9} L_{\odot}\right)$} & \multicolumn{3}{|c|}{ PAH Equivalent Width ${ }^{\mathrm{a}}(\mu \mathrm{m})$} & \multicolumn{3}{|c|}{$L_{\mathrm{IR}}^{\mathrm{b}}\left(10^{12} L_{\odot}\right)$} \\
\hline & $6.2 \mu \mathrm{m}$ & $7.7 \mu \mathrm{m}$ & $8.6 \mu \mathrm{m}$ & $6.2 \mu \mathrm{m}$ & $7.7 \mu \mathrm{m}$ & $8.6 \mu \mathrm{m}$ & SB & AGN & Total \\
\hline AzLOCK.62 & $19.4 \pm 1.1$ & $82.7 \pm 2.3$ & $16.8 \pm 4.6$ & $0.62 \pm 2.30$ & $3.43 \pm 11.44$ & $0.77 \pm 2.48$ & 3.2 & 1.8 & 5.0 \\
\hline AzLOCK.01 & $76.5 \pm 24.5$ & $387.1 \pm 11.1$ & $64.4=$ & $0.26 \pm 0.22$ & $1.80 \pm 1.68$ & $0.34 \pm 0.33$ & 7.9 & 13.6 & 21.5 \\
\hline SXDF850.02 & $15.8 \pm 9.4$ & $161.9 \pm 8.0$ & $22.9 \pm 11.5$ & $0.62 \pm 5.03$ & $6.61 \pm 37.52$ & $0.96 \pm 4.50$ & 8.3 & 1.6 & 10.0 \\
\hline AzLOCK.10 & $30.1 \pm 14.9$ & $168.4 \pm 28.7$ & $26.4 \pm 11.2$ & $0.23 \pm 0.14$ & $1.59 \pm 0.84$ & $0.27 \pm 0.14$ & 5.5 & 5.9 & 11.3 \\
\hline LOCK850.15 & $26.6 \pm 6.5$ & $125.2 \pm 4.2$ & $36.3 \pm 4.5$ & $0.26 \pm 0.17$ & $1.23 \pm 0.52$ & $0.36 \pm 0.13$ & 10.7 & 4.2 & 14.9 \\
\hline SXDF850.37 & $12.6 \pm 8.1$ & $86.8 \pm 4.3$ & $8.9 \pm 8.5$ & $0.42 \pm 0.88$ & $4.63 \pm 12.19$ & $0.54 \pm 1.52$ & 3.6 & 1.6 & 5.2 \\
\hline AzLOCK.05 & $36.5 \pm 8.7$ & $193.0 \pm 8.0$ & $2.8 \pm 4.5$ & $1.21 \pm 8.38$ & $6.58 \pm 31.27$ & $0.10 \pm 0.37$ & 7.8 & 2.3 & 10.1 \\
\hline LOCK850.01 & $25.7 \pm 5.0$ & $96.9 \pm 31.9$ & & $0.27 \pm 0.50$ & $0.59 \pm 0.44$ & & 6.5 & 3.3 & 9.8 \\
\hline
\end{tabular}

Notes.

${ }^{a}$ The equivalent widths are all in the rest frame.

${ }^{\mathrm{b}}$ Obtained from the best-fitting linear combination of an SB component (the SMG starburst composite SED from P08 comprised of their SMG SB composite spectrum spliced with the best-fitting modified CE01 template) and an AGN component (a Mrk 231 template) to the IRS spectrum and 850 or $1100 \mu \mathrm{m}$ photometry (see the text). The errors are typically $\lesssim 10 \%$ on each component.

relative fractions and luminosities of the $\mathrm{SB}\left(L_{\mathrm{SB}}\right)$ and $\mathrm{AGN}$ ( $\left.L_{\mathrm{AGN}}\right)$ components to the total infrared luminosity (see Table 4 ). Note that the full SF-SMG composite SED from P08 already contains a 30\% AGN contribution in the mid-infrared (from 5 to $11.5 \mu \mathrm{m}$ in the rest frame), which is taken into account in our calculations of $L_{\mathrm{AGN}}$ and $L_{\mathrm{SB}}$.

After the level of AGN contamination in each SMG has been determined in this way, we calculate the star formation rates (SFRs) for our objects from $L_{\mathrm{SB}}$ following Kennicutt (1998), assuming a starburst less than $100 \mathrm{Myr}$ in age with a Salpeter (1955) initial mass function.

\section{RESULTS}

We now present the results of our experiment, including the source spectra and redshifts (Section 3.1), the spectral decomposition and a comparison with the X-ray view in order to gather a complete census of the energetics of the sample (Section 3.2), the construction of a composite AGN-dominated SMG (Section 3.3), and finally the full SED fits (Section 3.4). We stress that we have followed similar recipes to those of P08 in order to facilitate a direct comparison with samples of more typical SF-dominated SMGs (see Section 2.4). 


\subsection{Source Spectra and Redshifts}

We present the final reduced spectra and fits in Figure 4. At a glance, the spectra reveal that our sample of SMGs possesses $\mathrm{PAH}$ features atop a range of mid-infrared continuum slopes. Despite the strong continuum in many of the spectra, measurable PAH features are apparent in all of our spectra, and we use these to extract redshifts (see Section 2.4.1 and Table 3). Near-infrared spectroscopic redshifts have recently been obtained for SXDF850.02 and SXDF850.37 (S. Alaghband-Zadeh et al. 2010, in preparation) of $z=2.518 \pm 0.001$ (showing narrow lines) and $z=2.769 \pm 0.001$ (showing broad $H \alpha$ and O III5007), respectively, in agreement with the IRS-derived redshifts. Finally, we note the suggestion by Ivison et al. (2005) that a galaxy at $z_{\mathrm{spec}}=2.15,3$ " west of LOCK850.1 may be associated with the SMG. Our Spitzer-IRS result suggests otherwise, and we discuss this source further in the Appendix.

The redshift distribution of our color-selected sample of eight objects ranges from $z=2.5$ to 3.4 , with a median of 2.76. This is noticeably shifted to higher redshifts compared to the P08 and MD09 samples, which have a median redshift of 2.0, which is more consistent with the known redshift distribution for radioidentified SMGs with $S_{850}>5 \mathrm{mJy}$ and $z \sim 2.2$ (Chapman et al. 2005). In Section 4.1, we suggest that the cause for this overall redshift difference between the sub-samples is likely due to selection effects.

\subsection{Spectral Decomposition of Individual Galaxies}

\subsubsection{Relative AGN/SF Contribution in the Mid-Infrared}

Using the fitting procedure described in Section 2.4.2 to determine the relative AGN/SF contribution in the mid-infrared, we have tabulated the continuum (AGN) fraction for each SMG in Table 3. We find a wide range of continuum fractions in our sample - from sub-dominant to significant_-with a median continuum contribution to the mid-infrared of $\approx 56 \%$. SXDF850.02 and SXDF850.37 have recently been spectroscopically confirmed at rest-frame optical wavelengths by $\mathrm{S}$. Alaghband-Zadeh et al. (2010, in preparation), and the rest-frame optical line widths are in broad agreement with our mid-infrared AGN fractions of $10 \%$ (where narrow lines suggest negligible AGN contribution) and $62 \%$ (where broad $H \alpha$ and $\mathrm{O}$ III5007 lines suggest the presence of an AGN), respectively. 5/8 (62\%) SMGs in our sample are clearly continuum-dominated sources in the mid-infrared ( $\gtrsim 50 \%$ of the mid-infrared luminosity). For comparison, only $2 / 13$ (15\%) SMGs from P08 have $>50 \%$ AGN contribution in the mid-infrared, and the median AGN contribution of their sample (including those with upper limits) is $\approx 34 \%$.

Although deeply embedded $\mathrm{H}$ II regions could potentially be contributing to the hot dust continuum that we here associate with an AGN contribution (making the fractions quoted above merely upper limits to the AGN contribution), we argue below that the dust heated by stars is likely a minor contribution. In Figure 5, we have plotted our 3.6-24 $\mu \mathrm{m}$ photometry in the rest-frame to search for any evidence of the $\mathrm{H}$-opacity minimum (1.6 $\mu \mathrm{m}$ stellar bump), as traced by the 4.5 and $5.8 \mu \mathrm{m}$ channels given our source redshifts, which is normally visible in typical SF SMGs (e.g., Hainline et al. 2009; S. J. Messenger et al. 2010, in preparation). The rest-frame broadband photometry of our sample of SMGs reveals a strong power-law shape from 1 to $3 \mu \mathrm{m}$. This exercise reveals that on average our sample of $S_{8} / S_{4.5}>2$ SMGs shows no $1.6 \mu \mathrm{m}$ stellar bump, whereas more typical SB-dominated SMGs $\left(S_{8} / S_{4.5}<2\right)$ do (see also

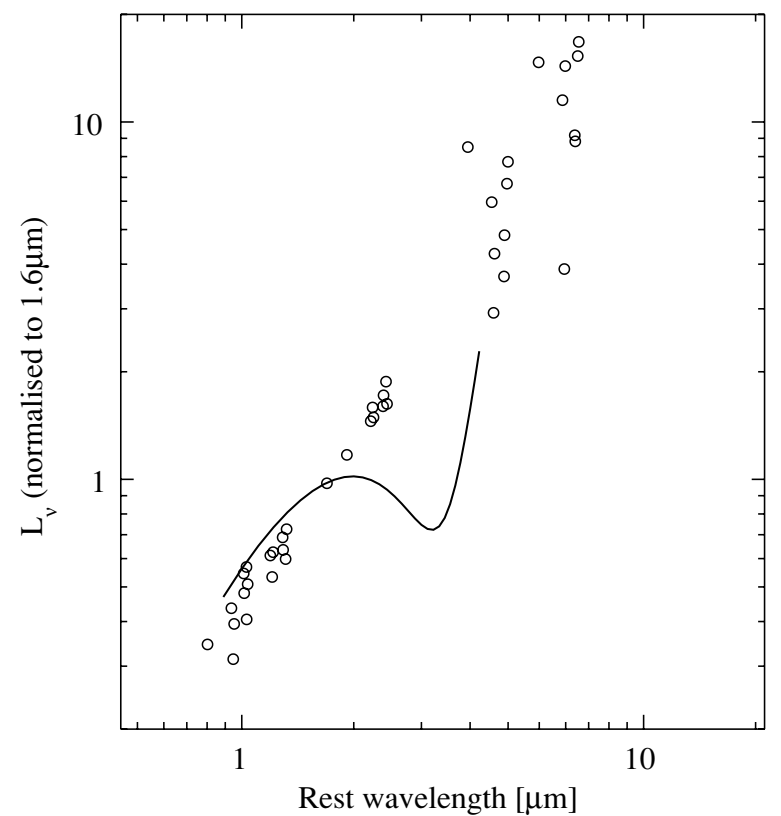

Figure 5. Rest-frame near-infrared SED of our sample of $S_{8} / S_{4.5}>2$ SMGs (open circles) compared with a fourth-order polynomial fitted to $S_{8} / S_{4.5}<2$ (SB-dominated) SMGs from S. J. Messenger et al. (2010, in preparation). This simple comparison shows that on average our sample of $S_{8} / S_{4.5}>2$ SMGs are dominated by power-law continuum emission which swamps the stellar emission bump normally seen in typical SB-dominated SMGs.

Yun et al. 2008). We thus interpret the $S_{8} / S_{4.5}>2$ observed color excess as due to hot dust emission $(500-1000 \mathrm{~K})$ in the immediate vicinity of an active nucleus (Laurent et al. 2000). By extension, this AGN emission would also dominate at the longer wavelengths probed by the IRS spectra $(\lesssim 10 \mu \mathrm{m})$, causing the power-law shape that we observe. Tentative evidence for AGN emission in these SMGs is apparent at radio wavelengths in the form of enhanced $1.4 \mathrm{GHz}$ emission compared to $610 \mathrm{MHz}$ data (see Section 3.4.1). We can directly test how likely it is that the AGN is producing the steep continuum emission in the mid-infrared spectra by examining the combination of the mid-infrared and X-ray data, as we now describe.

Using the mid-infrared continuum fits from Section 2.4.2, we calculate rest-frame $6 \mu \mathrm{m}$ luminosities due to the AGN component and compare these with the X-ray luminosities (Table 3). Comparing the X-ray and mid-infrared data can potentially tell us about the intrinsic luminosity of the AGNs and can reveal whether any of the sources are Compton-thick by comparing the estimated column densities to other samples of SMGs and local AGNs. Following Alexander et al. (2008b), in Figure 6 we have plotted the rest-frame $2-10 \mathrm{keV}$ versus $6 \mu \mathrm{m}$ luminosities for our sample compared to AGNs in the local universe and to other SMGs. Assuming the X-ray-to-midinfrared luminosity relationship for local AGNs (Lutz et al. 2004) holds at high redshift, the combination of the intrinsic Xray luminosities and $6 \mu \mathrm{m}$ luminosities of some of our objects suggests that they may be Compton-thick (i.e., four have upper limits close to the lower dotted line in Figure 6). While the X-ray data are not sufficiently deep (cf. 2 Ms Chandra data in GOODS-N, e.g., Alexander et al. 2008b) to conclusively determine whether or not the AGNs are Compton-thick, the mid-infrared spectra show that AGNs are dominating in these sources (in the form of strong continuum emission), and the non-detections are consistent with sources with being absorbed at X-ray energies (i.e., $N_{\mathrm{H}} \gtrsim 10^{22} \mathrm{~cm}^{-2}$ ). Indeed since $7 / 8$ 


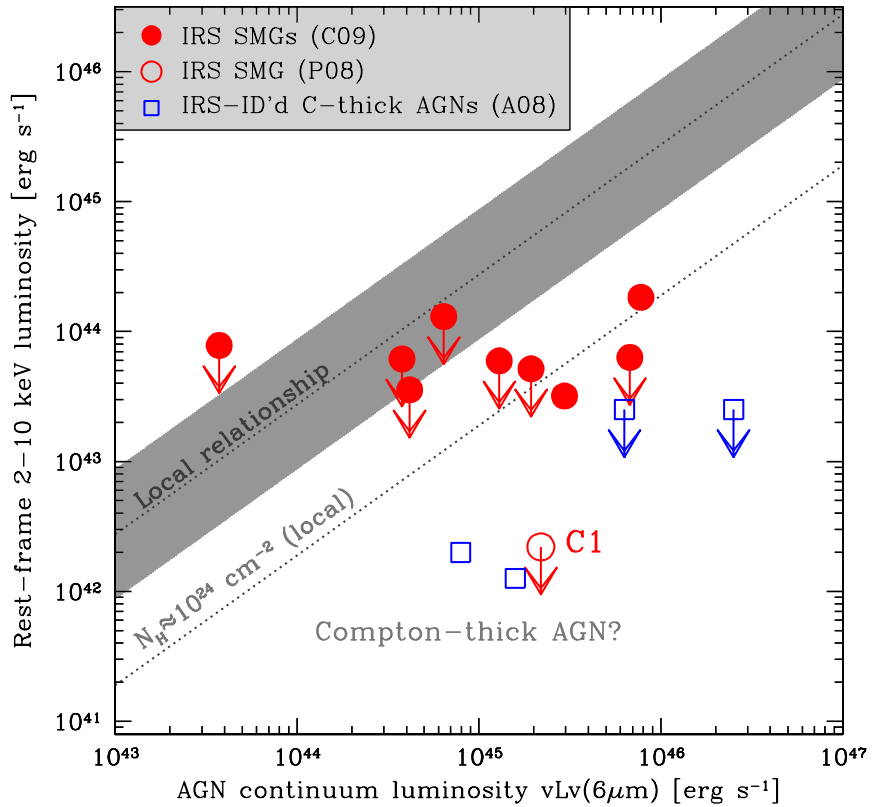

Figure 6. Rest-frame $2-10 \mathrm{keV}$ luminosity derived from the X-ray vs. $6 \mu \mathrm{m}$ luminosity derived from the mid-infrared power-law component fit (filled circles, where arrows indicate X-ray $3 \sigma$ upper limits). For comparison, we overplot Spitzer-IRS $z \sim 2$ AGNs with optical spectroscopy from Alexander et al. (2008b; open squares) and the SMG C1 from P08 (open circle) which have properties suggesting they are Compton-thick. We have also overplotted the intrinsic (absorption corrected) X-ray-to-mid-infrared luminosity relationship for local AGNs (Lutz et al. 2004; the shaded region) and, assuming that it holds at high redshift, the combination of the observed (not corrected for absorption) $\mathrm{X}$-ray luminosities and $6 \mu \mathrm{m}$ luminosities of some of our objects suggests that they could be Compton-thick (see Figure 4 of Alexander et al. 2008b; the lower dotted line refers to the observed luminosity ratio expected for a typical AGN that is absorbed by $N_{\mathrm{H}} \simeq 10^{24} \mathrm{~cm}^{-2}$ using the model presented in Alexander et al. 2005a). Note that $\mathrm{C} 1$ from P08 is also continuum-dominated in the midinfrared, but it possesses a much lower $L_{X}$ upper limit since it lies in a much deeper X-ray field.

(A color version of this figure is available in the online journal.)

SMGs are also optically faint with $R>23$, the majority of the sample appear to be obscured to some degree. Compared to other SMGs, most of our objects are consistent with typical moderate-luminosity AGNs in ULIRGs/SMGs, while two of our objects (AzLOCK.01 and AzLOCK.10) are more consistent with extreme AGN-dominated types.

\subsubsection{Mid-infrared Continuum (AGN) properties}

In light of the results in Section 3.2.1, we assume from now on that the continuum components of the fits are due to AGN activity, and we compare the mid-infrared AGN properties of our sample with more typical SB-dominated SMGs. In general, the continuum portion of our fits from Section 2.4 reveals that our SMGs display more strongly rising mid-infrared continuum when compared to more typical PAH-dominated SMGs (which comprise $\sim 80 \%$ of the SMG population; P08; MD09). In addition, while we do not see strong evidence for silicate absorption at $\sim 9.7 \mu \mathrm{m}$ in our IRS spectra (which is often seen in an AGN-dominated source), it cannot be ruled out since the $\mathrm{S} / \mathrm{N}$ of the continuum between 9 and $10 \mu \mathrm{m}$ is not high enough. Notably, all of the best-fitting models to our sample require some level of "additional" continuum component compared to typical SMGs. Unfortunately, the data are of relatively low S/N and possess insufficient wavelength coverage to provide robust measurements of individual object spectral slopes. Nevertheless, for completeness, we find a range of $\alpha=0-4.6$ (uncorrected for extinction, and assuming the convention $S_{v} \propto v^{-\alpha}$ ) with a mean and median of 1.6 and 1.8, respectively. In contrast, MD09 report a median $\alpha$ of 1.05 for their radio-detected sample of (mostly) PAH-dominated SMGs. In addition, P08 found that $8 / 13$ of their sample of more typical SB-dominated SMGs require a negligible level of continuum emission (and more often none) compared to the PAH template that was fitted.

\subsubsection{PAH Properties}

Here we present the properties of the PAH features measured in Section 2.4.1 (see Table 4), a key signature of luminous SF galaxies. We have tried to achieve the best PAH line measurements possible for this sample in order to compare our results with those of more typical SB-dominated samples of SMGs, and to other galaxy populations such as ULIRGs and AGNs, in order to yield insight into the physics of these systems. However, many of our PAHs are swamped by continuum emission and so this makes a fair comparison with other samples difficult, given the intrinsic differences in the spectra, the systematic differences in the way lines are measured for the different sub-samples (e.g., how extinction is taken into account), and the large measurement uncertainties in the derived quantities (see, e.g., P08 for a discussion). Nevertheless, qualitatively we find that within the systematic uncertainties, our $6.2 \mu \mathrm{m}$ PAH luminosities ${ }^{27}$ (median $2.7 \times 10^{10} L_{\odot}$ ) are toward the top end of SB-dominated SMGs studied by P08 and MD09, who find a spread covering about $\sim 0.5-3.5 \times 10^{10} L_{\odot}$ in their samples. The errors on the EWs are currently too uncertain to determine if there is a genuine spread in the EW of SB/SMGs compared with our sample, although we see the trend noted by P08 and MD09 that the SMGs in our sample with the largest AGN fractions have the smallest 6.2 and $7.7 \mu \mathrm{m}$ EWs. Next, we discuss the similarities and differences in the bulk PAH properties between this sample and others by constructing a composite spectrum of our AGN-dominated SMGs (see Section 3.3).

\subsection{Composite for AGN-dominated SMGs}

The spectral decomposition has revealed that some of the SMGs in our sample look more similar to those in P08, with low AGN contributions to the mid-infrared, while some of our SMGs clearly look more like the rarer AGN-dominated Compton-thick objects like $\mathrm{C} 1$ from $\mathrm{P} 08$. In order to compare the ensemble of properties of AGN-dominated versus SF-dominated SMGs and other galaxy populations, we have combined the IRS spectra of AGN-dominated ( $>50 \%$ continuum contribution in the midinfrared) SMGs from all available samples and created a higher $\mathrm{S} / \mathrm{N}$ composite spectrum. Using this composite we can search for and characterize faint features.

The composite is comprised of 10 SMGs including our five mid-infrared AGN-dominated SMGs, C1 (82\%) and GN04 (61\%) from P08, as well as SMMJ105238.30, SMMJ123553.26, and SMMJ163650.43 from MD09. ${ }^{28}$ Since the individual spectra span a wide dynamic range in rest-frame luminosity, we normalized them all to the median $\sim 7 \mu \mathrm{m}$ luminosity of the

\footnotetext{
27 We limit our comparison of PAH line fluxes to the $6.2 \mu \mathrm{m}$ feature since the $7.7 \mu \mathrm{m}$ feature is blended with other PAH features $(8.6 \mu \mathrm{m}$, for example) and the $9.7 \mu \mathrm{m}$ extinction trough which further complicates comparisons between different samples.

28 Since MD09 do not explicitly provide individual mid-infrared AGN fractions, we have re-derived them here, following the procedure in Section 2.4.2, and find AGN fractions of 94, 85, and 69\%, respectively, fulfilling our "AGN-dominated" criterion.
} 


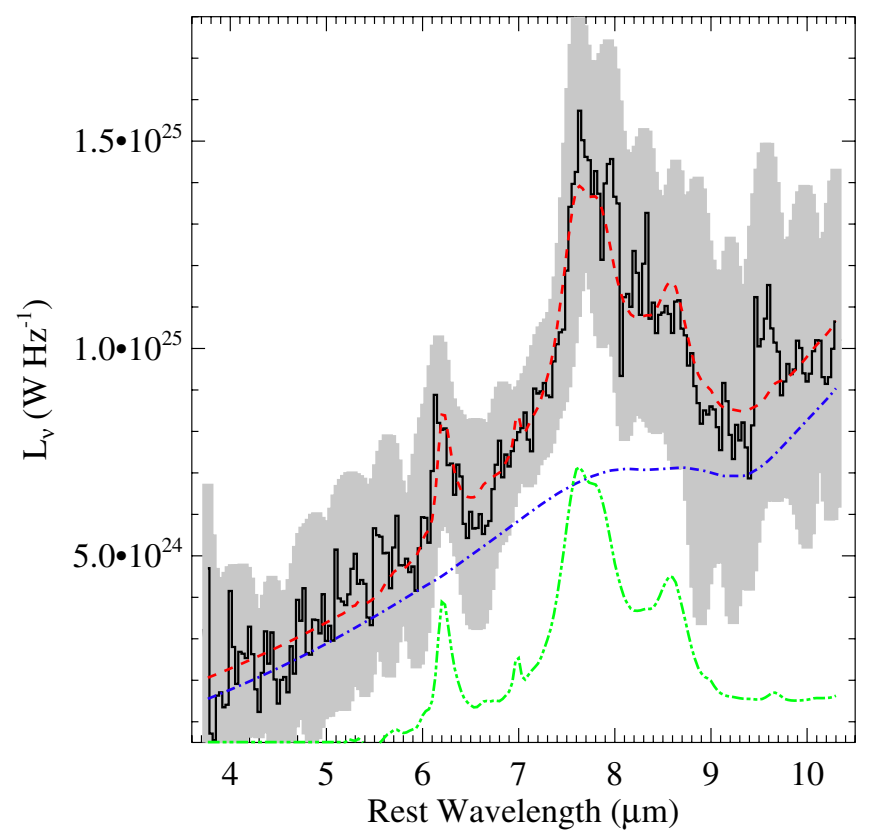

Figure 7. Composite IRS spectrum of 10 mid-infrared AGN-dominated SMGs (see the text). The shaded region shows the $1 \sigma$ uncertainty. The red dashed line is the best-fitting model (see Section 2.4.2), which is composed of a PAH template (green triple-dot-dashed line) and a power-law component with extinction applied (blue dot-dashed line), revealing a 72\% AGN fraction and an unabsorbed power-law component slope of $\simeq 2$ (see Section 3.3).

sample. ${ }^{29}$ We calculate the standard deviation of the sample as a measure of the error on the composite spectrum, which dominates over the uncertainty from individual flux elements. We restrict the final SMG composite spectrum wavelength coverage to areas where there are $>3$ data files, resulting in a coverage of $\sim 4-10 \mu \mathrm{m}$ in the rest-frame (see Figure 7).

We perform a spectral decomposition on the AGN composite, following the method outlined in Section 2.4.2, revealing a 72\% AGN fraction and an unabsorbed power-law component slope of $\simeq 2$. The amount of extinction required is $\tau_{9.7} \simeq 0.5$, but is not well constrained (as mentioned previously) since the spectrum does not extend much past $9.7 \mu \mathrm{m}$ (but by eye, it seems that the slope is flatter due to extinction at longer wavelengths).

In Figure 8, we compare our mid-infrared AGN-dominated SMG composite to the more typical PAH-dominated SMG composite of P08 and to a composite of $z \sim 2$ bright $\left(S_{24}>\right.$ $0.9 \mathrm{mJy}$ ) red $24 \mu \mathrm{m}$-selected ULIRGs from Sajina et al. (2007), which are mainly weak-PAH emitters and AGN-dominated sources. First of all, Figure 8 demonstrates that our AGNdominated composite is $\simeq 5$ times brighter at $\sim 7 \mu \mathrm{m}$ than the SB-dominated SMG composite, and we have not renormalized them to the same luminosity in order to highlight this difference. Given that the observed mid-infrared spectra and fluxes are so similar between these two samples, about a factor of $\simeq 1.5-2$ can be accounted for simply by the apparently higher average redshift of the AGN-dominated combined sample (median $z=2.56$ ) compared with the SF-dominated SMG sample (median $z=1.98$ ), and we discuss the possibility of selection effects further in Section 4. Figure 8 reveals that on average these SMGs have proportionately stronger/steeper mid-infrared continuum emission than more typical SF SMGs, as well as PAH

\footnotetext{
29 We normalize the spectra to the median total luminosity between rest frame 6.5 and $7.5 \mu \mathrm{m}$, where there is a lack of PAH features or absorption, in order to minimize the potential bias.
}

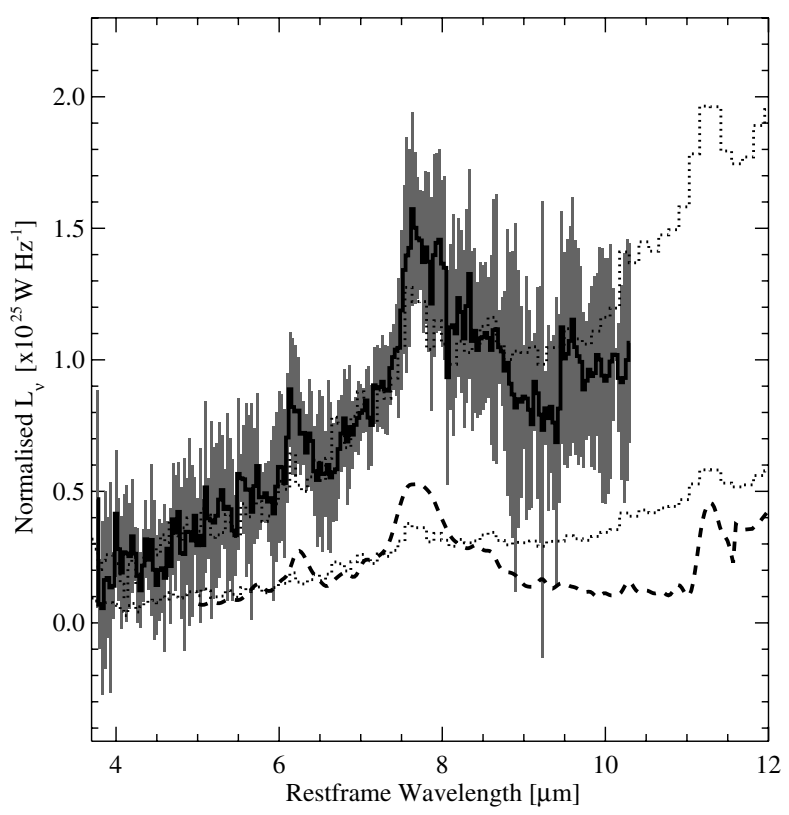

Figure 8. Composite IRS spectrum of 10 mid-infrared AGN-dominated SMGs (black histogram, with the shaded region representing the $1 \sigma$ uncertainty). We have overlaid the SB-dominated SMG composite from P08 (dashed curve), and note that the AGN-dominated SMG composite is $\simeq 5$ times more luminous at $\sim 7 \mu \mathrm{m}$ than the SB-dominated SMG composite). We have also overplotted the $\tau_{9.7}<1$ weak PAH composite of $1724-\mu \mathrm{m}$-selected ULIRGs (dotted histograms) from Sajina et al. (2007) twice, once renormalized at $\sim 7 \mu \mathrm{m}$ to match the AGN-dominated composite and also separately to match the SB-dominated composite, in order to highlight the relative similarities and differences between the three composites. This comparison reveals that AGNdominated SMGs appear to have features broadly in between that of SMGs and ULIRGs.

emission at 6.2, 7.7, and $8.6 \mu \mathrm{m}$, as well as a hint of silicate absorption at $\sim 9.7 \mu \mathrm{m}$ (although the data are very noisy in this region of the spectrum). At $>9-10 \mu \mathrm{m}$ the average AGNdominated SMG is more similar to the low opacity $\left(\tau_{9.7}<1\right)$ $24 \mu \mathrm{m}$-selected ULIRGs from Sajina et al. (2007) than to the SMG composite. In summary, in terms of their mid-infrared spectra, AGN-dominated SMGs broadly appear to have features somewhere in between SMGs (strong SF activity indicated by PAH emission) and $24-\mu \mathrm{m}$-selected ULIRGs (ongoing SF with a relatively strong AGN component).

\subsection{Full SED Fits}

We have established mid-infrared AGN dominance for the majority of our SMG sample. However, it is not clear if the AGN activity will continue to produce a dominant fraction of the total infrared luminosity, since it is expected that the AGN will become less important bolometrically than SBs when extrapolating from the mid-infrared to longer wavelengths (Tran et al. 2001; P08). Here we investigate the effect of the AGN emission to the total infrared luminosity when longer wavelength (submm) emission near the peak of the bolometric energy output of the galaxy is taken into account.

To begin with, we fit the mid-infrared spectrum and submm/ $\mathrm{mm}$ data (but not the radio data; see Section 3.4.1) for each source with the full SF-SMG composite SED from P08, which describes typical SMGs well (and includes a $\simeq 30 \%$ AGN contribution). Visually, all of the fits look poor and unsurprisingly have values of $\chi_{\text {red }}^{2} \gg 1$, meaning that the assumed model does not provide a very good description of the data. Since we already have evidence from the mid-infrared 

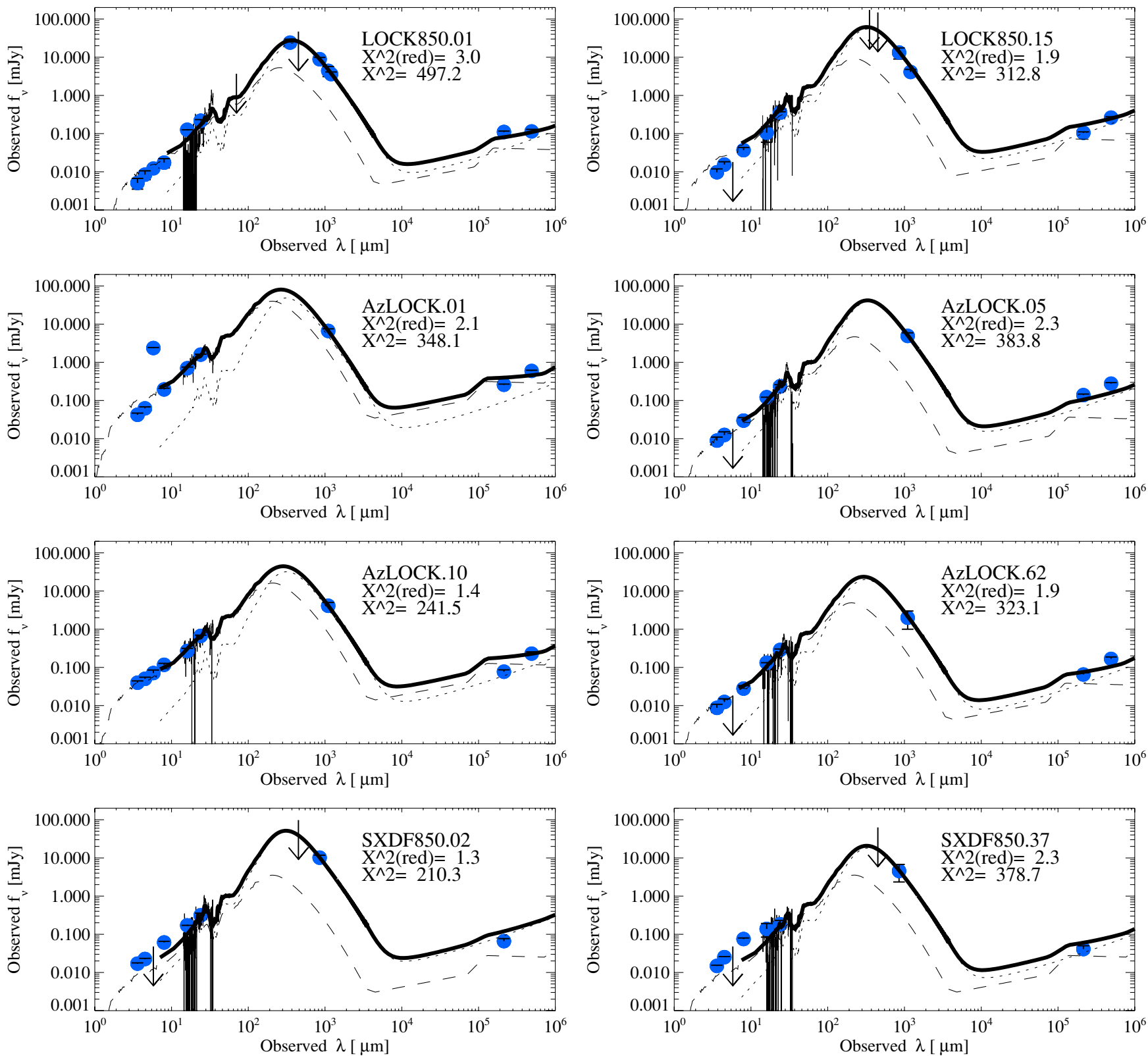

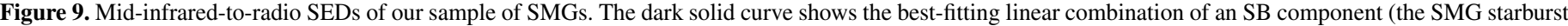

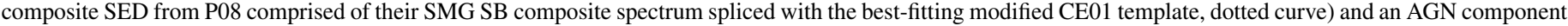

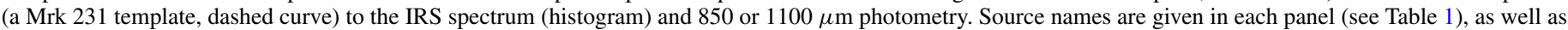

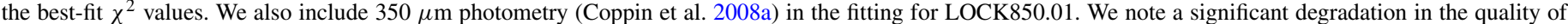

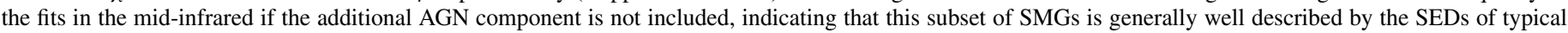

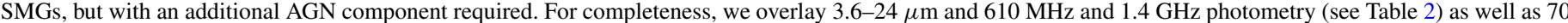

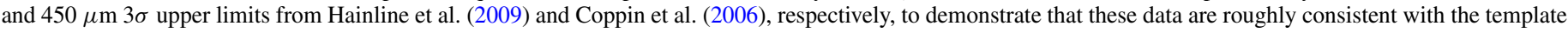
fitting, even though they are not used explicitly.

fitting in Section 3.2.1 supporting a significant continuum/ AGN contribution over typical SMGs in many cases, we repeat the fitting with a linear combination of the SF-SMG composite and an additional AGN component represented by Mrk 231, as described in Section 2.4.3. Visually the fits improve drastically, and we find an improvement in $\chi_{\text {red }}^{2}$ values in every case, typically of $\Delta \chi_{\text {red }}^{2} \simeq 3$ (though as large as $\sim 10$ for AzLOCK.10 and $\sim 60$ for AzLOCK.01), supporting the inclusion of the additional AGN template into the total SED model (see Figure 9).

A wider range of templates and/or models could be explored in principle; however, many complications could arise and make the results difficult to interpret. Since we wish to compare our decomposition directly with that of more typical SB-dominated SMGs (P08), the most natural choice for the AGN component is the SED of Mrk 231 since this is also what P08 used. Mrk 231 is a well-studied local ULIRG with strong evidence for an emerging AGN. For example, Genzel et al. (1998) conclude from high-excitation line measurements that Mrk 231 is likely to be powered mainly by an AGN, and more recently, Armus et al. (2007) find a mid-infrared AGN fraction of $~ 100 \%$. Although Mrk 231 appears to be AGN-dominated in several wavebands, it could also harbor some amount of dusty SF which would bias our bolometric AGN fractions upward. In order to verify this 
possibility, we repeat the SED fitting with the nuclear spectrum of the well-known local AGN NGC 1068 (R. Chary 2009, private communication; Genzel et al. 1998; Lutz et al. 2000) in place of Mrk 231 and, although the relative shapes of their dust spectra indicate that NGC 1068 is cooler than Mrk 231, the fitting yields the same AGN fractions to within a few percent. We also explore SF/AGN combinations using M82 as the SB component (instead of the P08 composite) and find that while it reproduces the mid-infrared PAH features well, M82 catastrophically fails to fit the submm/mm photometry, as expected since M82 has a much warmer dust temperature than SMGs. A more extensive comparison using a suite of many-parameter models (e.g., Siebenmorgen \& Krügel 2007) is beyond the scope of our simple approach given the sparsity of the data, although could be attempted in the near future as the data quality and SED coverage could be improved with upcoming Herschel and JWST surveys, for example.

Using these fits, we have calculated values of total $L_{\mathrm{IR}}$ as well as $L_{\mathrm{SB}}$ and $L_{\mathrm{AGN}}$ for the SF and AGN components separately and give these in Table 4 (see Section 2.4.3). The fractional contribution of AGNs to the total $L_{\mathrm{IR}}$ in our sample ranges from $17 \%$ to $63 \%$, with evidence for only two SMGs being bolometrically dominated by an AGN (>50\% maximal AGN contribution-AzLOCK.01 and AzLOCK.10 — which also have the strongest AGN components in the mid-infrared). Combining our five SMGs with a $>50 \%$ AGN contribution with $\mathrm{C} 1$ and GN04 from P08, the average AGN contribution to the bolometric luminosity, $L_{\mathrm{IR}}$, is $\simeq 40 \%$, compared with $15 \%$ for typical SFdominated SMGs from P08. We thus find a median $L_{\mathrm{SB}}$ for our sample of $6.5 \times 10^{12} L_{\odot}$, which translates into a median SFR for the sample of $\simeq 1000 M_{\odot} \mathrm{yr}^{-1}$, similar to typical bright SBdominated SMGs with $5 \times 10^{12} L_{\odot}$ (Pope et al. 2008a). Our sample thus has a similar $L_{\mathrm{IR}}$ to typical SB-dominated SMGs (only $\sim 1.5$ times more compared to P08), and thus it appears that our SMGs are from a similar SB luminosity class with similar SFRs, but that our sample has proportionately higher AGN content in the mid-infrared. We discuss the implications of these results and how they might fit into an evolutionary context with other SMGs in Section 4.

We have only fit the SEDs to the mid-infrared spectra and the submm data, but note that extrapolation of the best-fitting SEDs does generally fit the observed IRAC and $610 \mathrm{MHz}$ photometry of our SMGs well, with some amount of scatter at $1.4 \mathrm{GHz}$ (see Figure 9). Radio frequencies of $1.4 \mathrm{GHz}$ are sensitive to synchrotron radiation from relativistic electrons from supernovae (and hence recent SF; Condon et al. 1992), although an AGN can also produce comparable synchrotron emission by jets and radio lobes, leading to ambiguities in the cause of the mechanism at $\mu \mathrm{Jy}$ levels in the absence of other information. Since we know that these SMGs are harboring AGNs, it is quite possible (and likely) that the cause for the disagreement between the SED fits and the $1.4 \mathrm{GHz}$ data is due to an AGN which we now investigate by examining their measured 1.4 GHz-610 MHz spectral indices for clues.

\subsubsection{Radio Spectral Indices}

Ibar et al. (2010) calculate $1.4 \mathrm{GHz}-610 \mathrm{MHz}$ radio spectral indices based on radio maps from Ibar et al. (2009) using a fixed-to-beamsize source extraction, and they find a mean $1.4 \mathrm{GHz}-610 \mathrm{MHz}$ radio spectral index of $\alpha_{610}^{1.4}=-0.75 \pm 0.06$ $\left(S_{v} \propto v^{\alpha}\right)$ for the bulk of SMGs in the LH, consistent with optically thin synchrotron emission. The spectral indices for those of our SMGs which lie in the $\mathrm{LH}$ have a range of $-1.32<\alpha<-0.05$ (see Table 2). Five of these LH SMGs have steep radio spectra, $\alpha_{610}^{1.4} \lesssim-0.84$, while one has a self-absorbed flat spectrum, all of which are clearly deviant from typical SMGs. This difference seen in this subset of SMGs is suggestive that the radio emission has a different origin in these systems. Since we know from the mid-infrared spectral signatures that these SMGs likely harbor AGNs, it seems reasonable to assume that the AGN is affecting the radio emission, although other environmental causes could be playing a role (Ibar et al. 2010). In order to quantify this we calculate the logarithmic ratio of the rest-frame infrared and radio luminosities for SMGs using the $q_{L}$ parameter from Kovács et al. (2006), and find a range of $q_{L}$ values from 2.0 to 2.5 , with a median value of $2.21 \pm 0.15$ compared to $2.14 \pm 0.07$ for radio-detected bright SMGs. Although there is no evidence for an offset in $q_{L}$ compared to Kovács et al. (2006), the spectral indices suggest that the radio emission could be contaminated by radio-quiet AGN emission, providing some justification for omitting these data from the fitting.

\section{DISCUSSION}

These new IRS spectra of AGN-dominated SMGs have helped to confirm the predictive power of the $S_{24} / S_{8.0}-S_{8.0} / S_{4.5}$ diagram. Our sample was selected to lie off the expected SB sequence in the diagnostic color-color plot (Figure 1), and we have confirmed that the continuum emission dominates over PAH emission in the mid-infrared for 5/8 of our sources, which we interpret as due to a powerful AGN. The majority of our SMGs possess stronger/steeper mid-infrared continuum emission than more typical SB-dominated SMGs, indicating that there is a continuous trend in AGN content in SMGs-from low-luminosity AGNs to extremely luminous AGNs. As with all color-selection methods, the IRAC color selection is merely a guide to singling out the most powerful AGNs, and some level of contamination from SB systems is to be expected. In particular, SF systems at $z \lesssim 1$ could have $S_{8} / S_{4.5}>2$, since the 4.5 and $8 \mu \mathrm{m}$ channels are not yet climbing up the restframe $1.6 \mu \mathrm{m}$ stellar bump. For example, the most discrepant data point on the AGN track is an SMG at $z=0.689$ from Hainline et al. (2009) which is clearly SB-dominated in the IRS spectrum (MD09). The color cut is thus appropriate for separating mid-infrared SB- and AGN-dominated SMGs from $z \simeq 1-4$. However, since most SMGs lie within $1<z<4$ (Chapman et al. 2005), negligible contamination is expected on either side of the color-cut boundary. In light of the IRS results of MD09, who observed a large fraction of the submm-bright radioselected sample of Chapman et al. (2005) which have optical spectroscopic redshifts, we advocate adjusting the selection slightly to $S_{8} / S_{4.5} \gtrsim 1.65$ to account for the AGN-dominated SMGs seen in the MD09 sample. Even then, we still see some small amount of scatter to either side of this line (see Figure 1). Combining all the IRS-observed blank-field SMG samples, we find that $\simeq 15 \%$ of SMGs are mid-infrared AGN-dominated systems. Our full SED fitting reveals that 2/8 (25\%) of SMGs in our sample with AGN-dominant mid-infrared emission are likely bolometrically dominated by an AGN (in the far-infrared). This implies that overall $\sim 5 \%$ of blank-field SMGs are likely to have a dominant contribution to their $L_{\mathrm{IR}}$ due to AGN activity.

Now that we have confirmed that the majority of our sample is comprised of star-forming SMGs with an excess of AGN emission compared to typical SMGs, we can interpret this result within the framework of the proposed evolutionary sequence 
which links our AGN-dominated sample of SMGs to typical SMGs. However, before placing these results in any wider context, we must first take stock of any potential selection effects and how they could bias our interpretation.

\subsection{Selection Effects}

The eight targets in our color-selected sample have $850 \mu \mathrm{m}$ and $24 \mu \mathrm{m}$ flux densities of $4-13 \mathrm{mJy}^{30}$ and $180-1600 \mu \mathrm{Jy}$, with median values of $9 \mathrm{mJy}$ and $310 \mu \mathrm{Jy}$, respectively (see Table 2). Note that our targets have a similar $24 \mu \mathrm{m}$ flux density distribution as the whole SHADES SMG sample (i.e., our color selection has not biased the sample to preferentially brighter or fainter $24 \mu \mathrm{m}$ SMG counterparts). Thus, our IRAC colorselected subset of SMGs studied here should be representative of $\sim 15 \%$ of the SMG population discovered in existing blank-field $850 \mu \mathrm{m}$ imaging. The primary submm/ $\mathrm{mm}$ selection uniformly identifies high-redshift galaxies with $L_{\mathrm{SB}}>10^{12} L_{\odot}$ and corresponding SFRs of $\sim 1000 M_{\odot} \mathrm{yr}^{-1}$. The AGN-dominated SMG sample we have focused on here is similar to typical SMGs in terms of $L_{\mathrm{SB}}$, which allows for a fair and direct comparison between the two sub-samples of SMGs. However, there is some evidence that our sample lies at a slightly higher average redshift than typical SMGs, which merits some discussion.

The redshift distribution of our color-selected sample of eight objects ranges from 2.5 to 3.4 , with a median of 2.8 . This is noticeably shifted to higher redshifts compared to the P08 and MD09 samples, which have a median redshift of 2.0, more consistent with the known redshift distribution for radioidentified SMGs with $S_{850}>5$ mJy $(z \sim 2.2$; Chapman et al. 2005). The bias in our redshift distribution to earlier epochs is likely a submm selection effect, which tends to pick out warmer galaxies on average at higher redshift (e.g., Pope et al. 2006; Coppin et al. 2008a; Dye et al. 2009). The subsequent IRAC color selection imposed could be effectively singling out those higher redshift SMGs which possess warm dusty tori heated by a proportionately larger AGN. This trend was also noted by MD09 and Watabe et al. (2009), who found that "typical" SMGs show an increase in mid-infrared continuum strength (and less prominent PAHs) with increasing redshift. One way to verify this trend is to measure the flux near the far-infrared dust peak of the SED in order to constrain the dust temperatures in our $S_{8} / S_{4.5}>2$ sample and compare them to the dust temperatures of more typical SMGs with $S_{8} / S_{4.5}<2$, over a wide redshift range. Facilities such as Herschel and SCUBA-2 will be able to help address this issue for this sample, although some progress could be made now using BLAST which has already measured the rest-frame IR peaks of hundreds of SMGs in other fields where Spitzer IRAC data exist (Devlin et al. 2009; Dye et al. 2009). However, it is unlikely that the average redshift difference (equivalent to $\simeq 1$ Gyr in cosmic time) will have a large effect on the main conclusions, since even SMGs at $z \sim 4-5$ seem to show a mix of starburst and AGN signatures/SB properties similar to SMGs at $z \sim 2$ (e.g., Coppin et al. 2009).

\subsection{Where do Our AGN-dominated SMGs Fit into the Evolutionary Sequence?}

For the purposes of this discussion, we adopt the proposed evolutionary sequence first proposed by Sanders et al. (1988) and test the scenario where the SMG population evolves through

\footnotetext{
30 Note that we have converted the AzTEC $1.1 \mathrm{~mm}$ fluxes to $850 \mu \mathrm{m}$ fluxes by multiplying by a factor of 1.8 , according to the average $S_{850} / S_{1100}$ color ratio observed for SMGs (e.g., Chapin et al. 2009).
}

a primarily starburst phase, onto a subsequent submm-detected AGN phase, into a submm-undetected QSO and finally a passive elliptical. Spitzer-IRS data have tentatively identified some local ULIRGs being in key stages in this life cycle (Farrah et al. 2009). Now we ask - are the observed properties of typical SMGs and the AGN-dominated SMG sample studied here consistent with this picture in the high-redshift universe in terms of their space densities and other observed properties?

$S_{850}>4.5 \mathrm{mJy}$ SMGs have surface densities of $\simeq 600 \mathrm{deg}^{-2}$ (Coppin et al. 2006) or space densities of $\simeq 10^{-5} \mathrm{Mpc}^{-3}$ for $z=$ 1-3 (Chapman et al. 2005; Wall et al. 2008). The combination of this IRS work and other samples of SMGs has uncovered a mid-infrared presence of appreciable heating of a dust torus by an AGN for approximately $15 \%$ of the SMG population at this epoch. Assuming that all bright SMGs go through a subsequent AGN phase then the observed IRS-confirmed AGN-detection fraction merely represents a "duty cycle." The current total lifetime estimates from modeling SMGs are $~ 100-300 \mathrm{Myr}$ (Swinbank et al. 2006), and thus the inferred submm-bright AGN-dominated phase caught by the mid-infrared would be occurring during the last $\sim 15-45 \mathrm{Myr}$ of an SMG lifetime. These relative lifetimes and duty cycle are consistent with the merger simulations of Springel et al. (2005), where the tidal forces from galaxy mergers trigger a nuclear SB, fuelling a rapid growth of BHs before the gas has been consumed. The $\mathrm{BH}$ accretion rate grows throughout the peak phase of SF in the merger system (with the peak phase lasting $\approx 100 \mathrm{Myr}$ in these simulations), peaking $\approx 50 \mathrm{Myr}$ later than the SFR peak due to the delayed action of AGN feedback on the gas and manifesting itself as a luminous QSO for $\approx 50 \mathrm{Myr}$ total. In the Springel et al. (2005) simulations, the SMG phase is, in principle, both an SB and an AGN, but it is only during the final evolutionary stage that the remnant would be visible as an AGN, with outflows removing the surrounding gas and dust which heavily obscures the AGN at the beginning of the burst. Tentative evidence for this has been found in typical SMGs since their estimated $M_{\mathrm{BH}} / M_{\mathrm{gal}}$ ratio is lower than found in $z \sim 2$ QSOs (Alexander et al. 2008a; Coppin et al. 2008b; Peng et al. 2006).

Recently, Dey et al. (2008) proposed that Dust Obscured Galaxies (DOGs; $S_{v}(24 \mu \mathrm{m}) / S_{v}(R) \gtrsim 1000$ and $S_{24}>$ $300 \mathrm{mJy}$, with surface densities similar to $>6 \mathrm{mJy}$ SMGs) could represent a brief evolutionary phase between SMGs and less obscured QSOs or galaxies, which is supported by the finding that $\sim 30 \%$ of SMGs (including the AGN-dominated SMGs) meet the DOG criteria (Pope et al. 2008b). Interestingly, we note that virtually all (5/7 with $R$-band coverage) of our targets here fulfill the DOG criteria, confirming that the majority of AGN-dominated SMGs also overlap with the DOG population.

While these arguments are mainly circumstantial, we have shown that the AGN-dominated SMGs at least fit into the evolutionary picture of Sanders et al. (1988) in terms of the inferred duty cycles. While the statistical approach to duty cycles and relative lifetimes can be useful, in-depth studies of supposedly individual SMGs often reveal a complicated picture involving multiple components (e.g., Ivison et al. 2008). Measuring gas masses with the IRAM Plateau de Bure Interferometer or the Atacama Large Millimeter Array, inferring gas depletion timescales, and estimating SMBH masses using near-infrared spectroscopy for the AGN-dominated sample studied here (see, e.g., Alexander et al. 2008a; Coppin et al. 2008b) would allow us to put further constraints on the SMG evolutionary sequence we are exploring. 


\section{CONCLUSIONS}

Spitzer-IRS spectroscopy has been obtained for a sample of eight SMGs brighter than $200 \mu \mathrm{Jy}$ at $24 \mu \mathrm{m}$ and selected to possess observed IRAC colors of $S_{8} / S_{4.5}>2$ in order to investigate the level of AGN dominance in the SMG population. Our conclusions are as follows.

1. Taking into account sources in the literature, we find a that a color selection of $S_{8} / S_{4.5}>1.65$ is a better description overall for defining the boundary between SB and AGNdominated SMGs, with a small amount of scatter across this division.

2. We see signs of both SF and AGN activity in our sample of SMGs, with a continuous distribution of AGN fractions in the mid-infrared. There are signs of SF in the form of PAH features in all of the SMGs in our sample, from which we derive redshifts between 2.5 and 3.4, demonstrating the power of the mid-infrared to determine redshifts when the optical counterparts are too faint to study with current facilities.

3. We find a median AGN contribution to the mid-infrared of $56 \%$ in our sample of SMGs, indicating that overall, SMGs with $S_{24}>0.2 \mathrm{mJy}$ and $S_{8} / S_{4.5}>2$ tend to have more dominant AGN components in the mid-infrared than typical SMGs ( $<30 \%$ mid-infrared AGN contribution). Extrapolation to the far-infrared reveals that the AGN is bolometrically important only in two of our SMGs (each with a $>75 \%$ mid-infrared AGN contribution). This result suggests that significant contamination from AGN to the far-infrared luminosities affects $\lesssim 5 \%$ of the SMG population overall.

4. To first order, these AGN range from being fairly lowluminosity types found in typical SMGs to more extreme cases that likely overlap with AGN-dominated 24- $\mu \mathrm{m}$ selected ULIRGs in Sajina et al. (2007). In any case, all our AGNs appear to be obscured to some degree (at least five have low $L_{\mathrm{X}} / L_{6 \mu \mathrm{m}}$ and six are optically faint with $R>23$ ) and a small subset of our sample may be obscured by Compton-thick material.

5. Our results are thus consistent with the Sanders et al. (1988) evolutionary scenario, with all SMGs undergoing a "transitional" AGN-dominated phase with a duty cycle of $\simeq 15 \%$. Our sample of AGN-dominated SMGs could be at a slightly later stage of evolution than SF-dominated SMG systems, with the SF still occurring but where the AGN has now begun to heat the dust appreciably in the SMG as the $\mathrm{BH}$ undergoes a period of rapid growth.

This work is based on observations made with the Spitzer Space Telescope, which is operated by the Jet Propulsion Laboratory (JPL), California Institute of Technology under a contract with NASA. The IRS was a collaborative venture between Cornell University and Ball Aerospace Corporation funded by NASA through the JPL and Ames Research Center. Support for this work was provided by NASA through an award issued by JPL/Caltech. This work is based in part on data obtained as part of the UKIRT Infrared Deep Sky Survey (UKIDSS). K.C. acknowledges support from a UK Science and Technology Facilities Council fellowship. A.P. acknowledges support provided by NASA through the Spitzer Space Telescope Fellowship Program, through a contract issued by the JPL, California Institute of Technology under a contract with NASA. K.M.D. is supported by an NSF Astronomy and Astrophysics
Postdoctoral Fellowship under award AST-0802399. D.M.A. thanks the Royal Society and the Philip Leverhulme fellowship prize for generous support. We thank an anonymous referee for suggestions which improved the paper. We also thank Ranga Chary for providing the full SED template of Mrk 231 and the nuclear region of NGC 1068, and to Laura Hainline and Jim Geach for useful discussions.

Facilities: XMM, JCMT, GMRT, VLA, Spitzer

\section{APPENDIX}

\section{NOTES ON INDIVIDUAL SOURCES}

\section{A.1. LOCK850.1}

LOCK850.01 is the brightest SCUBA source detected in the LH region of the 8 mJy Survey (Scott et al. 2002; LE850.01) and SHADES (Coppin et al. 2006) and is also one of the brightest $1200 \mu \mathrm{m}$ sources detected in the Greve et al. (2004) MAMBO survey (LH1200.005). Lutz et al. (2001) identify extended $K$-band emission with the PdBI $1.2 \mathrm{~mm}$ confirmed position, and using the combination of redshift estimates based on the photometric properties of the extremely red object (ERO) counterpart they provide a "best guess" of $z=3$. Further spectroscopic follow-up of LOCK850.01 was obtained through optical and near-infrared spectroscopy (Simpson et al. 2004; Chapman et al. 2003, 2005; Blain et al. 2004; Ivison et al. 2005), although no secure redshift was forthcoming from the optically faint ERO. A galaxy $\sim 3^{\prime \prime}$ away from the radio counterpart was also targeted and estimated to lie at $z=2.148$ based on absorption lines in the LRIS spectrum. This redshift was tentatively assigned to LOCK850.01, since the likelihood of finding a $z \sim 2$ galaxy so close to the SMG centroid by chance is slim and so was deemed likely to be associated with the optically faint ERO (see Ivison et al. 2005 for a complete discussion). Based on different photometric redshift techniques Aretxaga et al. (2007) and Dye et al. (2008) find $z_{\text {phot }}=2.4_{0.2}^{1.1}$ (with a $90 \%$ confidence range of $2.2-3.8$ ) and $z_{\text {phot }}=4.21$ (with a $90 \%$ confidence range of $2.41-4.45$ ), respectively. Based on the presence of two PAHs in Figure $4, z=3.38 \pm 0.02$ is the $\chi^{2}$ best-fit solution, and we adopt this redshift for LOCK850.01.

\section{A.2. $L O C K 850.41-2$}

LOCK850.41 was first discovered in the $8 \mathrm{mJy}$ Survey (Scott et al. 2002; LE850.08) and by the Greve et al. (2004) $1200 \mu \mathrm{m}$ MAMBO survey (LH1200.014), and was reconfirmed by SHADES with an $850 \mu \mathrm{m}$ flux of $3.9_{-1.0}^{+0.9} \mathrm{mJy}$ (deboosted) and a submm position of R.A. $=10: 51: 59.86$, decl. $=57: 24: 23.6$ (Coppin et al. 2006). Ivison et al. (2005, 2007) and Chapman et al. (2005) located a statistically robust double counterpart in the radio for this system, as well as two coincidental $24 \mu \mathrm{m}$ counterparts, and obtained a convincing redshift of $z=0.689$ for the brightest, but furthest, of the counterparts. A spectrum for the other radio counterpart was also obtained, containing broadly similar spectral features but at much lower $\mathrm{S} / \mathrm{N}$ and so a redshift could not be unambiguously determined. The redshift of the $z=0.689$ counterpart (LOCK850.41-1) was confirmed by MD09 using the Spitzer-IRS. To complete the picture we obtained an IRS spectrum of LOCK850.41-2. This source originally followed our IRAC color selection criteria, although with a proper deblending of the IRAC photometry, it was found not to make our color selection cut after all, and so we have excluded this source from the main results and discussion of the paper. In Figure 10, the source appears to be completely 


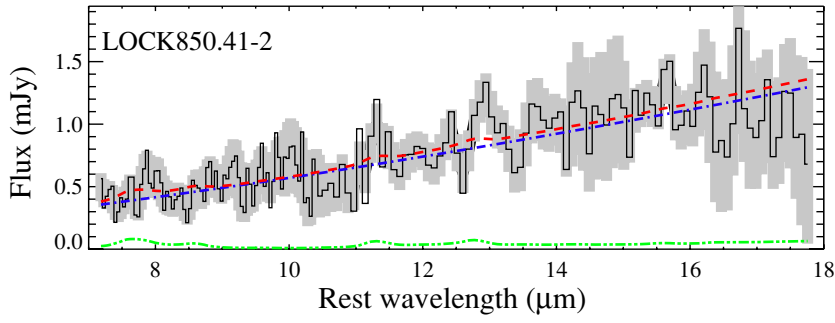

Figure 10. IRS spectrum of LOCK850.41-2: a continuum-dominated source in the mid-infrared ( $<90 \%$ continuum/AGN fraction) with no visible PAH features from which a redshift could be obtained. Instead we assume a source redshift of $z=0.974$, as indicated by the optical spectrum of the X-ray counterpart of the SMG from Lehmann et al. (2001). The solid black histogram is the raw (unsmoothed) IRS spectrum, while the shaded region represents the associated $1 \sigma$ noise from the sky background (see Section 2.3). The red dashed line is the best-fitting model composed of an M82 PAH template (green triple-dot-dashed line) and a power-law component with extinction applied (blue dot-dashed line). See Section 2.4.2.

continuum-dominated, with no strong discernable PAH features from which to determine a redshift. We find a formal "minimum" $\chi^{2}$ best fit at $z=3.1$ (see Section 2.4.1) if the weak feature seen at $\sim 11 \mu \mathrm{m}$ is assumed to be the $6.2 \mu \mathrm{m}$ PAH feature, although we note that this solution is not unique as all the reduced $\chi^{2}$ values are $<1$ for the $0.5<z<4.5$ range that was considered. This counterpart possesses a hard X-ray identification $\sim 2$ ".9 away $\left(S_{\mathrm{X}(0.5-2 \mathrm{keV})}=17.5 \times 10^{-16} \mathrm{erg} \mathrm{cm}^{-2} \mathrm{~s}^{-1} ; L_{\mathrm{X}(2-10 \mathrm{keV})}=\right.$ $18.3 \times 10^{43} \mathrm{erg} \mathrm{s}^{-1}$; see Figure 2) which is spectroscopically classified as a Type $1 \mathrm{AGN}$ in the optical, which could explain the lack of visible strong PAH features in the IRS spectrum at $z=0.974$ (Lehmann et al. 2001). Mainieri et al. (2002) find no evidence for X-ray absorption in this source, but do find a surprisingly flat $X$-ray spectral slope of $\Gamma \simeq 0.7$, compared with a typical $\Gamma \simeq 1.9$ for Type 1 AGNs. Despite the potential redshift ambiguity for LOCK850.41-2, the source is continuumdominated in the mid-infrared, regardless of the redshift.

\section{REFERENCES}

Alexander, D. M., Bauer, F. E., Chapman, S. C., Smail, I., Blain, A. W., Brandt, W. N., \& Ivison, R. J. 2005a, ApJ, 632, 736

Alexander, D. M., Brandt, W. N., Hornschemeier, A. E., Garmire, G. P., Schneider, D. P., Bauer, F. E., \& Griffiths, R. E. 2001, AJ, 122, 2156

Alexander, D. M., Smail, I., Bauer, F. E., Chapman, S. C., Blain, A. W., Brandt, W. N., \& Ivison, R. J. 2005b, Nature, 434, 738

Alexander, D. M., et al. 2008a, AJ, 135, 1968

Alexander, D. M., et al. 2008b, ApJ, 687, 835

Archibald, et al. 2002, MNRAS, 336, 353

Aretxaga, I., et al. 2007, MNRAS, 379, 1571

Armus, L., et al. 2007, ApJ, 656, 148

Ashby, M. L. N., et al. 2006, ApJ, 644, 778

Austermann, J. E., et al. 2010, MNRAS, 401, 160

Blain, A. W., et al. 2004, ApJ, 611, 725

Borys, C., Smail, I., Chapman, S. C., Blain, A. W., Alexander, D. M., \& Ivison, R. J. 2005, ApJ, 635, 853

Brandl, B. R., et al. 2006, ApJ, 653, 1129

Brunner, H., Cappelluti, N., Hasinger, G., Barcons, X., Fabian, A. C., Mainieri, V., \& Szokoly, G. 2008, A\&A, 479, 283

Chapin, E. L., et al. 2009, MNRAS, 398, 1793

Chapman, S. C., Blain, A. W., Ivison, R. J., \& Smail, I. R. 2003, Nature, 422 695

Chapman, S. C., Blain, A. W., Smail, I., \& Ivison, R. J. 2005, ApJ, 622, 772

Chary, R., \& Elbaz, D. 2001, ApJ, 556, 56

Clements, D. L., et al. 2008, MNRAS, 387, 247

Condon, J. J. 1992, ARA\&A, 30, 575

Coppin, K., et al. 2006, MNRAS, 372, 1621

Coppin, K., et al. 2008a, MNRAS, 384, 1597

Coppin, K., et al. 2008b, MNRAS, 389, 45

Coppin, K., et al. 2009, MNRAS, 395, 1905

Dasyra, K. M., et al. 2009, ApJ, 701, 1123

Devlin, M. J., et al. 2009, Nature, 458, 737
Dey, A., et al. 2008, ApJ, 677, 943

Draine, B. T. 2003, ARA\&A, 41, 241

Draine, B. T., \& Li, A. 2007, ApJ, 657, 810

Dye, S., et al. 2008, MNRAS, 386, 1107

Dye, S., et al. 2009, ApJ, 703, 285

Eales, S., et al. 2009, ApJ, 707, 1779

Farrah, D., et al. 2008, ApJ, 677, 957

Farrah, D., et al. 2009, ApJ, 700, 395

Fazio, G. G., et al. 2004, ApJS, 154, 10

Förster Schreiber, N. M., et al. 2003, A\&A, 399, 833

Frayer, D. T., Ivison, R. J., Scoville, N. Z., Yun, M., Evans, A. S., Smail, I., Blain, A. W., \& Kneib, J.-P. 1998, ApJ, 506, L7

Furusawa, H., et al. 2008, ApJS, 176, 1

Genzel, R., et al. 1998, ApJ, 498, 579

Greve, T. R., et al. 2004, MNRAS, 354, 779

Greve, T. R., et al. 2005, MNRAS, 359, 1165

Hainline, L. J., Blain, A. W., Smail, I., Frayer, D. T., Chapman, S. C., Ivison, R. J., \& Alexander, D. M. 2009, ApJ, 699, 1610

Hernán-Caballero, A., et al. 2009, MNRAS, 395, 1695

Holland, W. S., et al. 1999, MNRAS, 303, 659

Houck, J. R., et al. 2004, ApJS, 154, 18

Ibar, E., Ivison, R. J., Best, P. N., Coppin, K., Pope, A., Smail, I., \& Dunlop, J. S. 2010, MNRAS, 401, L53

Ibar, E., Ivison, R. J., Biggs, A. D., Lal, D. V., Best, P. N., \& Green, D. A 2009, MNRAS, 397, 281

Ivison, R. J., et al. 2004, ApJS, 154, 124

Ivison, R. J., et al. 2005, MNRAS, 364, 1025

Ivison, R. J., et al. 2007, MNRAS, 380, 199

Ivison, R. J., et al. 2008, MNRAS, 390, 1117

Kennicutt, R. C. 1998, ARA\&A, 36, 189

Kovács, A., Chapman, S. C., Dowell, C. D., Blain, A. W., Ivison, R. J., Smail, I., \& Phillips, T. G. 2006, ApJ, 650, 592

Laird, E. S., Nandra, K., Pope, A., \& Scott, D. 2010, MNRAS, 401, 2763

Laurent, O., et al. 2000, A\&A, 359, 887

Lawrence, A., et al. 2007, MNRAS, 379, 1599

Lehmann, I., et al. 2001, A\&A, 371, 833

Lilly, S. J., et al. 1999, ApJ, 518, 641

Lonsdale, C. J., et al. 2004, ApJS, 154, 54

Lutz, D., Maiolino, R., Spoon, H. W. W., \& Moorwood, A. F. M. 2004, A\&A, 418, 465

Lutz, D., Valiante, E., Sturm, E., Genzel, R., Tacconi, L. J., Lehnert, M. D., Sternberg, A., \& Baker, A. J. 2005, ApJ, 625, L83

Lutz, D., et al. 2000, ApJ, 536, 697

Lutz, D., et al. 2001, A\&A, 378, 70

Mainieri, V., Bergeron, J., Hasinger, G., Lehmann, I., Rosati, P., Schmidt, M. Szokoly, G., \& Della Ceca, R. 2002, A\&A, 393, 425

Menéndez-Delmestre, K., et al. 2007, ApJ, 655, L65

Menéndez-Delmestre, K., et al. 2009, ApJ, 699, 667 (MD09)

Mortier, A. M. J., et al. 2005, MNRAS, 363, 563

Murphy, E. J., Chary, R.-R., Alexander, D. M., Dickinson, M., Magnelli, B., Morrison, G., Pope, A., \& Teplitz, H. I. 2009, ApJ, 698, 1380

Peng, C. Y., et al. 2006, ApJ, 649, 616

Pope, A., et al. 2006, MNRAS, 370, 1185

Pope, A., et al. 2008a, ApJ, 675, 1171 (P08)

Pope, A., et al. 2008b, ApJ, 689, 127

Rieke, G. H., et al. 2004, ApJS, 154, 25

Rigopoulou, D., Spoon, H. W. W., Genzel, R., Lutz, D., Moorwood, A. F. M., \& Tran, Q. D. 1999, AJ, 118, 2625

Sajina, A., Yan, L., Armus, L., Choi, P., Fadda, D., Helou, G., \& Spoon, H. 2007, ApJ, 664, 713

Salpeter, E. E. 1955, ApJ, 121, 161

Sanders, D. B., Soifer, B. T., Elias, J. H., Neugebauer, G., \& Matthews, K. 1988, ApJ, 328, L35

Scott, S. E., et al. 2002, MNRAS, 331, 817

Siebenmorgen, R., \& Krügel, E. 2007, A\&A, 461, 445

Simpson, C., Dunlop, J. S., Eales, S. A., Ivison, R. J., Scott, S. E., Lilly, S. J., \& Webb, T. M. A. 2004, MNRAS, 353, 179

Skrutskie, M. F., et al. 2006, AJ, 131, 1163

Spergel, D. N., et al. 2003, ApJS, 148, 175

Springel, V., Di Matteo, T., \& Hernquist, L. 2005, MNRAS, 361, 776

Stevens, J. A., Page, M. J., Ivison, R. J., Carrera, F. J., Mittaz, J. P. D., Smail, I., \& McHardy, I. M. 2005, MNRAS, 360, 610

Swinbank, A. M., Chapman, S. C., Smail, I., Lindner, C., Borys, C., Blain, A. W., Ivison, R. J., \& Lewis, G. F. 2006, MNRAS, 371, 465

Swinbank, A. M., Smail, I., Chapman, S. C., Blain, A. W., Ivison, R. J., \& Keel, W. C. 2004, ApJ, 617, 64

Tacconi, L. J., et al. 2006, ApJ, 640, 228 
Tacconi, L. J., et al. 2008, ApJ, 680, 246

Takata, T., Sekiguchi, K., Smail, I., Chapman, S. C., Geach, J. E., Swinbank, A. M., Blain, A., \& Ivison, R. J. 2006, ApJ, 651, 713

Teplitz, H. I., et al. 2007, ApJ, 659, 941

Tran, Q. D., et al. 2001, ApJ, 552, 527

Ueda, Y., et al. 2008, ApJS, 179, 124

Valiante, E., et al. 2007, ApJ, 660, 1060
Wall, J. V., Pope, A., \& Scott, D. 2008, MNRAS, 383, 435

Watabe, Y., Risaliti, G., Salvati, M., Nardini, E., Sani, E., \& Marconi, A. 2009, MNRAS, 396, L1

Weedman, D. W., et al. 2006, ApJ, 653, 101

Wilson, G. W., et al. 2008, MNRAS, 386, 807

Yan, L., et al. 2007, ApJ, 658, 778

Yun, M. S., et al. 2008, MNRAS, 389, 333 